\title{
Particle Filtering for Nonlinear/Non-Gaussian Systems with Energy Harvesting Sensors Subject to Randomly Occurring Sensor Saturations
}

\author{
Weihao Song, Zidong Wang, Jianan Wang, Fuad E. Alsaadi and Jiayuan Shan
}

\begin{abstract}
In this paper, the particle filtering problem is investigated for a class of nonlinear/non-Gaussian systems with energy harvesting sensors subject to randomly occurring sensor saturations (ROSSs). The random occurrences of the sensor saturations are characterized by a series of Bernoulli distributed stochastic variables with known probability distributions. The energy harvesting sensor transmits its measurement output to the remote filter only when the current energy level is sufficient, where the transmission probability of the measurement is recursively calculated by using the probability distribution of the sensor energy level. The effects of the ROSSs and the possible measurement losses induced by insufficient energies are fully considered in the design of filtering scheme, and an explicit expression of the likelihood function is derived. Finally, the numerical simulation examples (including a benchmark example for nonlinear filtering and the applications in moving target tracking problem) are provided to demonstrate the feasibility and effectiveness of the proposed particle filtering algorithm.
\end{abstract}

Index Terms-Particle filtering, nonlinear/non-Gaussian systems, randomly occurring sensor saturations, energy harvesting sensor, multi-sensor systems

\section{INTRODUCTION}

As one of the fundamental issues in signal processing and control communities, the filtering problem has attracted considerable research interest during the past few decades [6], [17], [25], [32], [51]. As early as in [15], a general filtering framework has been constructed from a Bayesian point of view, where the probability density function (PDF) of the state of interest conditioned on the measurements has been computed in a recursive manner. For linear systems with Gaussian noises, it is well known that the renowned Kalman filter [33] offers the analytically optimal solution. For general nonlinear and/or non-Gaussian systems, the corresponding optimal solution exists only in a conceptual sense because

The Deanship of Scientific Research (DSR) at King Abdulaziz University, Jeddah, Saudi Arabia funded this project, under grant no. (FP-23-42). This work was also supported in part by the National Natural Science Foundation of China under Grant 61873031, 61873148, and 61933007, the China Scholarship Council under Grant 201806030206, and the Alexander von Humboldt Foundation of Germany. (Corresponding author: Jianan Wang.)

W. Song, J. Wang and J. Shan are with the School of Aerospace Engineering, Beijing Institute of Technology, Beijing 100081, China. (Email: wangjianan@bit.edu.cn)

Z. Wang is with the Department of Computer Science, Brunel University London, Uxbridge, Middlesex, UB8 3PH, United Kingdom. (Email: Zidong.Wang@brunel.ac.uk)

F. E. Alsaadi is with the Department of Electrical and Computer Engineering, Faculty of Engineering, King Abdulaziz University, Jeddah 21589, Saudi Arabia. the recursive propagation demands the calculation of multidimensional integrals that is numerically difficult. To solve this problem, various filtering algorithms have been developed by utilizing different numerical approximation methods with examples including, but are not limited to, extended Kalman filtering with first-order linearization [1], [31], unscented Kalman filtering with unscented transform [20], [21], cubature Kalman filtering with cubature rule [2], and particle filtering with Monte Carlo approximation [3], [12], [28], [61].

Owing to the applicability to general systems with any type of noises, the particle filtering algorithm has captured much attention from both academy and industry, and a rich body of results has been reported from various perspectives, such as reduced communication burden [27], model uncertainty [55] and packet dropouts [62]. Among others, the group importance sampling algorithm has been proposed in [40], which aims to compress the statistical information included in a set of weighted samples into a single summary weighted particle. In [42], a particle selection method has been proposed for cost-reference particle filtering algorithm, which is capable of overcoming the difficulty (incurred by resampling) of parallel computation in conventional particle filters. In addition, the particle filtering problem has been investigated in [45] considering the presence of out-of-sequence measurements.

In many real-world applications of networked systems, it is ubiquitous that the physical sensor cannot produce measurement signals with unlimited amplitude due mainly to the hardware restrictions, and this is referred to as the sensor saturation phenomenon. Such a phenomenon, if not properly considered in the stage of filter design, is likely to result in performance degradation or even algorithm divergence. Accordingly, a large amount of research attention has been devoted to the filtering problem with sensor saturations [11], [16], [37], [38], [47]. For example, the distributed set-membership filters have been designed for systems with sensor saturations, unknown but bounded noises and sector-bounded nonlinearities under the event-triggered communication scheme in [37]. The joint state and fault estimation problem has been investigated with variance constraints in [16] for the time-varying nonlinear systems subject to sensor saturations, parameter uncertainties and randomly occurring faults.

Recently, the phenomenon of randomly occurring sensor saturations (ROSSs) has started to draw some research attention, see e.g. [8], [58]. In a networked environment, due to limited bandwidth and/or unpredictable disturbances, the network itself might undergo data collisions leading to net- 
work congestions and subsequent network-induced behaviors (e.g. communication delays and packet dropouts [24], [52], [53]) which, in turn, give rise to the so-called ROSSs [63]. Note that the ROSSs can also result from abrupt environment changes and random/intermittent sensor failures [58]. Up to now, the filtering problem for systems suffering from ROSSs has aroused an initial yet quickly increasing research interest, see [26], [34], [56] and the references therein. Nevertheless, it is worth mentioning that the corresponding filter design issue for general non-Gaussian systems subject to ROSSs has not been investigated adequately.

It is often the case in practice that the energy supply is rather scarce and this inevitably imposes certain constraints on the efficient/smooth usage of the wireless sensor networks since more sensors are battery-operated. To prolong the sensors' lifetime, two kinds of approaches have been put forward, one is to reduce the energy consumption and the other is to increase the energy supply. For the former approach, considering that the wireless transmission of data streams constitutes the main cause for energy consuming, a great deal of work (e.g. [27], [35], [57], [59]) has been concerned with the event-triggered schemes where the data transmission is activated only when certain predefined event occurs. For the latter approach, there has been a notably growing research interest towards the so-called energy harvesting technology [14], [44], [54]. For sensors equipped with energy harvesters, the energy can be harnessed (and then replenished) from the surrounding environment such as solar and mechanical vibrations [23].

Given the practical importance of the energy harvesting sensors, the corresponding filter design problem has recently been gaining some initial attention. For example, in [22], the optimal energy allocation problem has been studied for multi-sensor estimation with energy harvesting and energy sharing technologies. In [18], both the state and the sensor energy level have been estimated for a linear Gaussian system by incorporating the available set-valued and point-valued event-triggered measurements. In [50], a recursion expression for the probability distribution of the energy level has been derived and an effective recursive filtering algorithm has been proposed to address the phenomenon of sensor-energy-induced missing measurements for a class of nonlinear time-delayed systems. It should be noted that, so far, most reported results have been focused on the linear Gaussian systems [18], [23] or the nonlinear systems with special nonlinearities [50]. The filter design issue for general nonlinear/non-Gaussian systems with energy harvesting sensors subject to ROSSs has not received adequate attention despite its practical significance.

Summarizing the above discussions, in this paper, we aim to deal with the particle filtering problem for a class of nonlinear/non-Gaussian systems involving both ROSSs and probabilistic missing measurements caused by the energy harvesting sensors. Through recursively calculating the probability distribution of the sensor energy level, the missing probability of the measurement is derived. Accordingly, a particle filtering algorithm is developed to restrain the effect from both the ROSSs and the limited sensor energy on the filtering performance by virtue of a modified likelihood function. The main contributions of this paper are highlighted as follows: 1) a unified framework for sequential Bayesian estimation is proposed for a general class of nonlinear/nonGaussian systems under the energy-dependent transmission protocol; 2) a modified particle filtering algorithm is developed to address the multi-sensor fusion problem subject to partial measurement-information-loss induced by different energy levels of each individual sensor; and 3) the recursion for importance weight is derived to take into account the joint effects from the ROSSs and the sensor energy constraints.

The remainder of this paper is organized as follows. In Section II, the concerned filtering problem is formulated. In Section III, the particle filtering algorithm is designed by considering the phenomenon of ROSSs and the energy harvesting sensors. In Section IV, two numerical examples are presented to show the usefulness and effectiveness of the proposed particle filtering scheme. Concluding remarks are finally provided in Section V.

Notation. Throughout this paper, the notation used is fairly standard. $\mathbb{R}^{n}$ represents the $n$-dimensional Euclidean vector space. $\|\cdot\|$ stands for the Euclidean norm of a vector. The superscript $T$ denotes the operation of transpose. $\operatorname{diag}\left\{a_{1}, a_{2}, \ldots, a_{n}\right\}$ denotes a diagonal matrix with $a_{1}, a_{2}, \ldots, a_{n}$ being the diagonal elements. $p_{x}(\cdot)$ stands for the PDF of a stochastic variable $x$, i.e., $x \sim p_{x}(\cdot)$, and $p_{x \mid y}(\cdot)$ denotes the conditional PDF of a stochastic variable $x$ given $y$. $\operatorname{Pr}\{A\}$ denotes the occurrence probability of the discrete event A. $N(a, b)$ represents the Gaussian distribution with mean $a$ and covariance $b . G(a, b)$ denotes the Gamma distribution with shape parameter $a$ and scale parameter $b \cdot \operatorname{Exp}(a)$ stands for the exponential distribution with mean $a . x_{k: l}$ represents the trajectory of $x$ from time instant $k$ to time instant $l$. Other notations will be provided as the need arises.

\section{Problem Formulation}

Consider the following discrete-time nonlinear system

$$
x_{k+1}=f_{k}\left(x_{k}\right)+\omega_{k}
$$

and $N$ energy harvesting sensors with randomly occurring saturations

$$
y_{k}^{i}=\gamma_{k}^{i} \operatorname{Sat}\left(h_{k}^{i}\left(x_{k}\right)\right)+\left(1-\gamma_{k}^{i}\right) h_{k}^{i}\left(x_{k}\right)+\nu_{k}^{i}
$$

where, for $i=1,2, \ldots, N, x_{k} \in \mathbb{R}^{n}$ and $y_{k}^{i} \in \mathbb{R}$ are the state of the target plant and the measurement output of the $i$ th sensor at time instant $k$, respectively. $f_{k}(\cdot): \mathbb{R}^{n} \mapsto \mathbb{R}^{n}$ and $h_{k}^{i}(\cdot): \mathbb{R}^{n} \mapsto \mathbb{R}$ denote the state transition function and the measurement function of the $i$ th sensor, respectively. $\omega_{k} \in \mathbb{R}^{n}$ represents the process noise satisfying $p_{\omega_{k}}(\cdot)$ and $\nu_{k}^{i} \in \mathbb{R}$ is the measurement noise on sensor $i$ satisfying $p_{\nu_{k}^{i}}(\cdot)$. The saturation function $\operatorname{Sat}(\cdot): \mathbb{R} \mapsto \mathbb{R}$ is modelled by

$$
\operatorname{Sat}(\kappa)=\operatorname{sign}(\kappa) \min \left\{\kappa_{\max },|\kappa|\right\}
$$

where $\operatorname{sign}(\cdot)$ is the signum function and $\kappa_{\max }$ represents the saturation level. Moreover, a Bernoulli distributed stochastic variable $\gamma_{k}^{i}$ is defined to characterize the phenomenon of the ROSSs on the $i$ th sensor [58], which takes a value of 0 or 1 with

$$
\left\{\begin{array}{l}
\operatorname{Pr}\left\{\gamma_{k}^{i}=1\right\}=\bar{\gamma}^{i} \\
\operatorname{Pr}\left\{\gamma_{k}^{i}=0\right\}=1-\bar{\gamma}^{i}
\end{array}\right.
$$


This article has been accepted for publication in a future issue of this journal, but has not been fully edited. Content may change prior to final publication. Citation information: DOI10.1109/TSP.2020.3042951, IEEE Transactions on Signal Processing

where $\bar{\gamma}^{i} \in[0,1]$ is a known constant.

Next, the following assumptions are made to further clarify the considered system.

Assumption 1: The initial state $x_{0}$ satisfies the prior density $p_{x_{0}}(\cdot)$, i.e., $x_{0} \sim p_{x_{0}}(\cdot)$.

Assumption 2: The process noise $\omega_{k}$, the $N$ measurement noises $\left\{\nu_{k}^{i}\right\}_{i=1}^{N}$ and the stochastic variables $\left\{\gamma_{k}^{i}\right\}_{i=1}^{N}$ are all mutually independent and also independent of the initial state $x_{0}$.

Assumption 3: The nonlinear functions $f_{k}(\cdot)$ and $h_{k}^{i}(\cdot)$ as well as the PDFs $p_{\omega_{k}}(\cdot)$ and $p_{\nu_{k}^{i}}(\cdot)$ are all known.

Let the $i$ th energy harvesting sensor be powered by a chargeable battery whose maximum energy storage capacity is denoted by $\bar{E}^{i}$ and the energy level of the $i$ th sensor at time instant $k$ is defined by $E_{k}^{i} \in\left\{0,1, \ldots, \bar{E}^{i}\right\}$. Denote $H_{k}^{i}$ as the number of the units of energy harvested by the $i$ th sensor at time instant $k$, which is modelled as a first-order Markov model. Assume that $H_{k}^{i}$ takes a value in the finite non-negative integer set $\mathbb{H}=\{0,1, \ldots, \bar{H}\}$ with transition probability matrix $\Pi=\left[\pi_{u v}\right]_{\bar{H} \times \bar{H}}$, where $\bar{H}$ denotes the maximum energy that can be harvested by the sensors. $\pi_{u v}=$ $\operatorname{Pr}\left\{H_{k+1}^{i}=v \mid H_{k}^{i}=u\right\}$ denotes the transition probability for all $u, v \in \mathbb{H}$ and $\sum_{v=0}^{\bar{H}} \pi_{u v}=1$.

Remark 1: As stated in [14], [23], the energy harvesting process may be correlated among different time instants, e.g. the amount of the harvested solar energy is contingent on the weather and the period of a day. The rationality of the first-order Markov energy harvesting model is justified by the empirical measurements when the solar energy is the energy harvesting source [13].

Assumption 4: The energy harvesting sensor can transmit its measurement to the remote filter only when it stores nonzero units of energy and each measurement transmission consumes one unit of energy.

Define an indicator variable $1_{\left\{E_{k}^{i}>0\right\}}$ for the energy consumption as

$$
1_{\left\{E_{k}^{i}>0\right\}}=\left\{\begin{array}{ll}
1, & \text { if } E_{k}^{i}>0 \\
0, & \text { otherwise }
\end{array},\right.
$$

then the energy level of the $i$ th sensor at each time instant is recursively calculated as [50]

$$
E_{k+1}^{i}=\min \left\{E_{k}^{i}-1_{\left\{E_{k}^{i}>0\right\}}+H_{k+1}^{i}, \bar{E}^{i}\right\}
$$

with the initial energy level $0<E_{0}^{i} \leq \bar{E}^{i}$. Note that the energy level is unknown at each time instant $k \geq 1$ due to the unpredictable characteristic of the amount of harvested energy, and the probability distribution of the energy level will be discussed in the subsequent section.

As illustrated in Fig. 1, the $i$ th energy harvesting sensor transmits the new measurement to the remote filter only when the sensor's current energy is sufficient, i.e., $E_{k}^{i}>0$. Due to the above-mentioned property of the energy harvesting sensors, at the remote filter side, the available measurement contributed by the $i$ th sensor at time instant $k$ is described as

$$
z_{k}^{i}=1_{\left\{E_{k}^{i}>0\right\}} y_{k}^{i}+n_{k}^{i},
$$

where $n_{k}^{i} \in \mathbb{R}$ denotes the channel noise in the reception of the $i$ th sensor's information, which satisfies $p_{n_{k}^{i}}(\cdot)$.

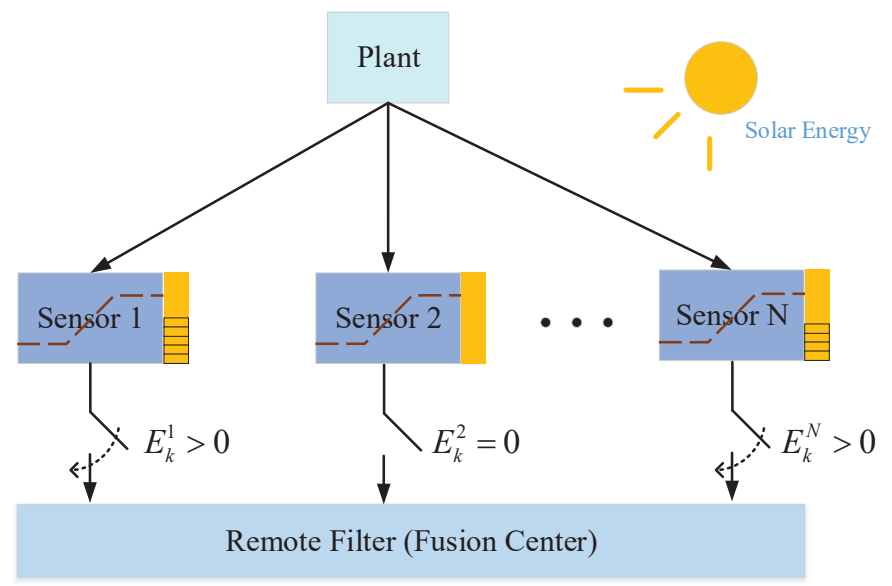

Fig. 1: Block diagram of the networked system with energy harvesting sensors and ROSSs.

Remark 2: Compared with the existing results concerning particle filtering with packet dropout [62] and $H_{\infty}$ filtering with missing measurements [49], two distinguishing features of the considered model are identified as follows. 1) A novel indicator variable $1_{\left\{E_{k}^{i}>0\right\}}$, whose probability distribution is dependent on the evolution dynamics of the energy level (6), is introduced to characterize whether or not the measurement $y_{k}^{i}$ is successfully transmitted to the remote filter. Note that $1_{\left\{E_{k}^{i}>0\right\}}$ is a time-correlated variable (rather than a Bernoulli distributed stochastic variable with known probability distribution). 2) Both the measurement noise and the channel noise are taken into consideration to better cater for the real-world scenario. In addition, there is no specific requirement on the type of the noise, and therefore the application potential is further increased. In summary, it is these distinguishing features that render some additional difficulties in the subsequent design of filtering scheme.

At time instant $k$, the available measurements at the remote filter are denoted as $z_{k}^{1: N}=\left[\begin{array}{llll}z_{k}^{1} & z_{k}^{2} & \cdots & z_{k}^{N}\end{array}\right]^{T}$. In addition, let us denote $Z_{1: k}^{1: N}=\left[\begin{array}{llll}\left(z_{1}^{1: N}\right)^{T} & \left(z_{2}^{1: N}\right)^{T} & \cdots & \left(z_{k}^{1: N}\right)^{T}\end{array}\right]^{T}$ as the vector of all available measurements up to time instant $k$.

The purpose of this paper is to develop a particle filtering algorithm for the general nonlinear/non-Gaussian systems subject to the sensor energy constraints and the ROSSs such that the estimate of state $x_{k}$ can be obtained at the remote filter in the sense of minimum mean-square error (MMSE) based on the available actual measurement information $Z_{1: k}^{1: N}$.

\section{Algorithm Design AND Discussion}

As is well known, the MMSE estimate of the unknown state vector $x_{k}$ is defined by

$$
\hat{x}_{k}=\int x_{k} p\left(x_{k} \mid Z_{1: k}^{1: N}\right) d x_{k} .
$$

Unfortunately, it is difficult to obtain an analytical solution to the problem addressed in this paper due to the fact that the marginal posterior PDF $p\left(x_{k} \mid Z_{1: k}^{1: N}\right)$ is non-Gaussian. As an alternative method, the particle filtering algorithm [3] can be 
utilized to obtain an approximate expression of $p\left(x_{0: k} \mid Z_{1: k}^{1: N}\right)$ with a set of weighted particle trajectories as

$$
p\left(x_{0: k} \mid Z_{1: k}^{1: N}\right)=\sum_{m=1}^{M} W_{k}^{m} \delta\left(x_{0: k}-x_{0: k}^{m}\right)
$$

where $M$ denotes the number of the particles and $\delta(\cdot)$ represents the multi-dimensional Dirac delta function. In addition, the particles $\left\{x_{0: k}^{m}\right\}_{m=1}^{M}$ are drawn from a proposal density function $q\left(x_{0: k} \mid Z_{1: k}^{1: N}\right)$ and the corresponding weight$\mathrm{s}\left\{W_{k}^{m}\right\}_{m=1}^{M}$ are calculated via the importance sampling method. To be more specific, the importance weight $W_{k}^{m}$ associated with $x_{0: k}^{m}$ is defined by

$$
W_{k}^{m}=\frac{p\left(x_{0: k}^{m}, Z_{1: k}^{1: N}\right)}{q\left(x_{0: k}^{m} \mid Z_{1: k}^{1: N}\right)} .
$$

If the proposal density function is selected such that we can factorize it as

$$
q\left(x_{0: k}^{m} \mid Z_{1: k}^{1: N}\right)=q\left(x_{k}^{m} \mid x_{0: k-1}^{m}, Z_{1: k}^{1: N}\right) q\left(x_{0: k-1}^{m} \mid Z_{1: k-1}^{1: N}\right),
$$

then we have

$$
\begin{aligned}
W_{k}^{m} & =\frac{p\left(x_{0: k}^{m}, Z_{1: k}^{1: N}\right)}{q\left(x_{0: k}^{m} \mid Z_{1: k}^{1: N}\right)} \\
& =\frac{p\left(z_{k}^{1: N} \mid x_{k}^{m}\right) p\left(x_{k}^{m} \mid x_{k-1}^{m}\right) p\left(x_{0: k-1}^{m}, Z_{1: k-1}^{1: N}\right)}{q\left(x_{k}^{m} \mid x_{0: k-1}^{m}, Z_{1: k}^{1: N}\right) q\left(x_{0: k-1}^{m} \mid Z_{1: k-1}^{1: N}\right)} .
\end{aligned}
$$

According to Assumption 2, it is straightforward to see that

$$
p\left(z_{k}^{1: N} \mid x_{k}^{m}\right)=\prod_{i=1}^{N} p\left(z_{k}^{i} \mid x_{k}^{m}\right) .
$$

Substituting (13) into (12) yields

$$
W_{k}^{m}=W_{k-1}^{m} \frac{\left(\prod_{i=1}^{N} p\left(z_{k}^{i} \mid x_{k}^{m}\right)\right) p\left(x_{k}^{m} \mid x_{k-1}^{m}\right)}{q\left(x_{k}^{m} \mid x_{0: k-1}^{m}, Z_{1: k}^{1: N}\right)} .
$$

It is evident from (2) and (7) that the actual measurement $z_{k}^{i}$ (contributed by the $i$ th energy harvesting sensor at the remote filter) is dependent on 1) the energy level and 2) whether the sensor saturation occurs or not. Therefore, the update equation of the importance weights, which is related to the indicator variable $1_{\left\{E_{k}^{i}>0\right\}}$ and the Bernoulli distributed stochastic variable $\gamma_{k}^{i}$, is distinct from that in the standard sequential importance sampling method. In the sequel, we aim to derive an explicit expression of the likelihood function with the purpose of compensating for the effects resulting from the ROSSs and the probabilistic missing measurements.

Proposition 1: With the measurement model described by (2) and (7), the likelihood function of the system state associated with the $i$ th energy harvesting sensor at time instant $k$ is given by

$$
\begin{aligned}
& p\left(z_{k}^{i} \mid x_{k}^{m}\right) \\
= & \operatorname{Pr}\left\{1_{\left\{E_{k}^{i}>0\right\}}=1\right\}\left[\left(1-\bar{\gamma}^{i}\right) p_{\nu_{k}^{i}+n_{k}^{i}}\left(z_{k}^{i}-h_{k}^{i}\left(x_{k}^{m}\right)\right)\right. \\
& \left.+\bar{\gamma}^{i} p_{\nu_{k}^{i}+n_{k}^{i}}\left(z_{k}^{i}-\operatorname{Sat}\left(h_{k}^{i}\left(x_{k}^{m}\right)\right)\right)\right] \\
& +\operatorname{Pr}\left\{1_{\left\{E_{k}^{i}>0\right\}}=0\right\} p_{n_{k}^{i}}\left(z_{k}^{i}\right)
\end{aligned}
$$

where $p_{\nu_{k}^{i}+n_{k}^{i}}(\cdot)$ is the PDF of the sum of the measurement noise $\nu_{k}^{i}$ and the channel noise $n_{k}^{i}$.
Proof: To prove the result, we will discuss the form of the likelihood function in the following three cases.

Case 1: If the energy of sensor node $i$ is sufficient and the phenomenon of sensor saturation does not occur at time instant $k$, i.e., $1_{\left\{E_{k}^{i}>0\right\}}=1$ and $\gamma_{k}^{i}=0$, it is immediate to see from (2) and (7) that

$$
z_{k}^{i}=h_{k}^{i}\left(x_{k}\right)+\nu_{k}^{i}+n_{k}^{i} .
$$

Then, the likelihood function is evaluated as

$$
p\left(z_{k}^{i} \mid x_{k}^{m}, 1_{\left\{E_{k}^{i}>0\right\}}=1, \gamma_{k}^{i}=0\right)=p_{\nu_{k}^{i}+n_{k}^{i}}\left(z_{k}^{i}-h_{k}^{i}\left(x_{k}^{m}\right)\right) .
$$

Case 2: If the energy of sensor node $i$ is sufficient and the phenomenon of sensor saturation occurs at time instant $k$, i.e., $1_{\left\{E_{k}^{i}>0\right\}}=1$ and $\gamma_{k}^{i}=1$, we obtain from (2) and (7) that

$$
z_{k}^{i}=\operatorname{Sat}\left(h_{k}^{i}\left(x_{k}\right)\right)+\nu_{k}^{i}+n_{k}^{i} .
$$

Then, we can denote the likelihood function as

$p\left(z_{k}^{i} \mid x_{k}^{m}, 1_{\left\{E_{k}^{i}>0\right\}}=1, \gamma_{k}^{i}=1\right)=p_{\nu_{k}^{i}+n_{k}^{i}}\left(z_{k}^{i}-\operatorname{Sat}\left(h_{k}^{i}\left(x_{k}^{m}\right)\right)\right)$.

Summarizing the above two cases, it is obtained from (17) and (19) that

$$
\begin{aligned}
& p\left(z_{k}^{i} \mid x_{k}^{m}, 1_{\left\{E_{k}^{i}>0\right\}}=1\right) \\
= & \sum_{j=0}^{1} p\left(z_{k}^{i}, \gamma_{k}^{i}=j \mid x_{k}^{m}, 1_{\left\{E_{k}^{i}>0\right\}}=1\right) \\
= & \sum_{j=0}^{1} p\left(z_{k}^{i} \mid x_{k}^{m}, 1_{\left\{E_{k}^{i}>0\right\}}=1, \gamma_{k}^{i}=j\right) \operatorname{Pr}\left\{\gamma_{k}^{i}=j\right\} \\
= & \left(1-\bar{\gamma}^{i}\right) p\left(z_{k}^{i} \mid x_{k}^{m}, 1_{\left\{E_{k}^{i}>0\right\}}=1, \gamma_{k}^{i}=0\right) \\
& +\bar{\gamma}^{i} p\left(z_{k}^{i} \mid x_{k}^{m}, 1_{\left\{E_{k}^{i}>0\right\}}=1, \gamma_{k}^{i}=1\right) \\
= & \left(1-\bar{\gamma}^{i}\right) p_{\nu_{k}^{i}+n_{k}^{i}}\left(z_{k}^{i}-h_{k}^{i}\left(x_{k}^{m}\right)\right) \\
& +\bar{\gamma}^{i} p_{\nu_{k}^{i}+n_{k}^{i}}\left(z_{k}^{i}-\operatorname{Sat}\left(h_{k}^{i}\left(x_{k}^{m}\right)\right)\right) .
\end{aligned}
$$

Case 3: If the energy of sensor node $i$ is insufficient and the current measurement is not transmitted at time instant $k$, i.e., $1_{\left\{E_{k}^{i}>0\right\}}=0$, then only the channel noise is received at the remote filter and we have

$$
z_{k}^{i}=n_{k}^{i} .
$$

Similarly, the likelihood function is rewritten as

$$
p\left(z_{k}^{i} \mid x_{k}^{m}, 1_{\left\{E_{k}^{i}>0\right\}}=0\right)=p_{n_{k}^{i}}\left(z_{k}^{i}\right) .
$$

By noting (20) and (22) together with the law of total probability, the likelihood function $p\left(z_{k}^{i} \mid x_{k}^{m}\right)$ with regard to the $i$ th sensor at time instant $k$ is expressed by

$$
\begin{aligned}
& p\left(z_{k}^{i} \mid x_{k}^{m}\right) \\
= & \sum_{j=0}^{1} p\left(z_{k}^{i}, 1_{\left\{E_{k}^{i}>0\right\}}=j \mid x_{k}^{m}\right) \\
= & \sum_{j=0}^{1} p\left(z_{k}^{i} \mid x_{k}^{m}, 1_{\left\{E_{k}^{i}>0\right\}}=j\right) \operatorname{Pr}\left\{1_{\left\{E_{k}^{i}>0\right\}}=j\right\} \\
= & \operatorname{Pr}\left\{1_{\left\{E_{k}^{i}>0\right\}}=1\right\} p\left(z_{k}^{i} \mid x_{k}^{m}, 1_{\left\{E_{k}^{i}>0\right\}}=1\right) \\
& +\operatorname{Pr}\left\{1_{\left\{E_{k}^{i}>0\right\}}=0\right\} p\left(z_{k}^{i} \mid x_{k}^{m}, 1_{\left\{E_{k}^{i}>0\right\}}=0\right)
\end{aligned}
$$




$$
\begin{aligned}
= & \operatorname{Pr}\left\{1_{\left\{E_{k}^{i}>0\right\}}=1\right\}\left[\left(1-\bar{\gamma}^{i}\right) p_{\nu_{k}^{i}+n_{k}^{i}}\left(z_{k}^{i}-h_{k}^{i}\left(x_{k}^{m}\right)\right)\right. \\
& \left.+\bar{\gamma}^{i} p_{\nu_{k}^{i}+n_{k}^{i}}\left(z_{k}^{i}-\operatorname{Sat}\left(h_{k}^{i}\left(x_{k}^{m}\right)\right)\right)\right] \\
& +\operatorname{Pr}\left\{1_{\left\{E_{k}^{i}>0\right\}}=0\right\} p_{n_{k}^{i}}\left(z_{k}^{i}\right),
\end{aligned}
$$

which completes the proof.

According to Proposition 1, the explicit expression of the likelihood function corresponding to the $i$ th energy harvesting sensor is now written as (15), which cannot be directly applied in the weight update due to the fact that the transmission probability $\operatorname{Pr}\left\{1_{\left\{E_{k}^{i}>0\right\}}=1\right\}$ is by far unknown. Before proceeding further, the following lemma is introduced to deal with such an issue.

Lemma 1: Given the dynamics of the energy level $\left\{E_{k}^{i}\right\}$ described by (6) with initial energy level $E_{0}^{i}$ and the firstorder Markov energy harvesting process $\left\{H_{k}^{i}\right\}$ with the initial distribution of $H_{0}^{i}$, the recursion of the probability distribution $\tau_{k}^{i}$ of the energy level for the $i$ th energy harvesting sensor can be calculated by

$$
\left\{\begin{array}{l}
p_{k, v}^{i}=\sum_{u=0}^{\bar{H}} \pi_{u v} p_{k-1, u}^{i} \\
\tau_{k}^{i}=\chi+S_{k}^{i} \tau_{k-1}^{i} \\
\tau_{0}^{i}=\left[\begin{array}{llll}
\underbrace{0}_{E_{0}^{i}} \cdots \quad 0 & 1 & \underbrace{0}_{\bar{E}^{i}-E_{0}^{i}} \cdots
\end{array}\right]^{T}
\end{array}\right.
$$

where

$$
\begin{aligned}
\tau_{k}^{i} & =\left[\begin{array}{lllll}
\operatorname{Pr}\left\{E_{k}^{i}=0\right\} & \cdots & \operatorname{Pr}\left\{E_{k}^{i}=\bar{E}^{i}\right\}
\end{array}\right]^{T}, \\
\chi & =\left[\begin{array}{ccccc}
0 \quad \cdots & 0 & 1
\end{array}\right]^{T}, \\
S_{k}^{i} & =\left[\begin{array}{ccccc}
p_{k, 0}^{i} & p_{k, 0}^{i} & 0 & \cdots & 0 \\
p_{k, 1}^{i} & p_{k, 1}^{i} & p_{k, 0}^{i} & \cdots & 0 \\
p_{k, 2}^{i} & p_{k, 2}^{i} & p_{k, 1}^{i} & \cdots & 0 \\
\vdots & \vdots & \vdots & \ddots & \vdots \\
p_{k, \bar{E}^{i}-1}^{i} & p_{k, \bar{E}^{i}-1}^{i} & p_{k, \bar{E}^{i}-2}^{i} & \cdots & p_{k, 0}^{i} \\
\bar{E}^{i}-1 & \bar{E}^{i}-1 & \bar{E}^{i}-2 & & \\
-\sum_{s=0}^{i} p_{k, s}^{i} & -\sum_{s=0}^{i} p_{k, s}^{i} & -\sum_{s=0}^{i} p_{k, s}^{i} & \cdots & -p_{k, 0}^{i}
\end{array}\right]
\end{aligned}
$$

and

$$
p_{k, v}^{i}=\operatorname{Pr}\left\{H_{k}^{i}=v\right\} .
$$

Proof: For $v \in \mathbb{H}$, according to the law of total probability, we have

$$
\begin{aligned}
p_{k, v}^{i} & =\operatorname{Pr}\left\{H_{k}^{i}=v\right\} \\
& =\sum_{u=0}^{\bar{H}} \operatorname{Pr}\left\{H_{k}^{i}=v, H_{k-1}^{i}=u\right\} \\
& =\sum_{u=0}^{\bar{H}} \operatorname{Pr}\left\{H_{k}^{i}=v \mid H_{k-1}^{i}=u\right\} \operatorname{Pr}\left\{H_{k-1}^{i}=u\right\} \\
& =\sum_{u=0}^{\bar{H}} \pi_{u v} p_{k-1, u}^{i},
\end{aligned}
$$

by which the probability distribution of the harvested energy quantity can be calculated at each time instant. The remaining part of this proof is similar to that of Lemma 1 in [50] and is omitted here.
From Lemma 1, we immediately obtain the transmission probability of the $i$ th sensor's measurement at time instant $k$, that is,

$$
\lambda_{k}^{i}=\operatorname{Pr}\left\{1_{\left\{E_{k}^{i}>0\right\}}=1\right\}=\left[\begin{array}{llll}
0 & \underbrace{1}_{\bar{E}^{i}} \cdots & \cdots
\end{array}\right] \tau_{k}^{i} .
$$

Remark 3: It should be noted that the structure of Lemma 1 is similar to that in [50], but the distinction lies in that the probability distribution of the harvested energy in Lemma 1 is time-varying and dependent on the previous time instant, which can be calculated recursively with a time-varying matrix $S_{k}^{i}$. In fact, Lemma 1 can be reduced to that in [50] if we simply set the probability transition matrix as an identity matrix. In addition, due to the limited hardware level of the energy harvesting modules, the maximum amount of energy that can be harvested by the $i$ th sensor might be less than the maximum amount that it can store, i.e., $\bar{H} \leq \bar{E}^{i}-1$. In this case, some elements in the matrix $S_{k}^{i}$ will be always equal to zeros, i.e., $p_{k, v}^{i}=0$ for $v>\bar{H}$. Meanwhile, the first $\bar{E}^{i}-\bar{H}+1$ elements of the last row in the matrix $S_{k}^{i}$ are equal to -1 by noting $\sum_{u=0}^{\bar{H}} p_{k, u}^{i}=1$. Consequently, the computational cost of the matrix $S_{k}^{i}$ will be reduced.

Now, substituting (15) and (26) into (13), we can evaluate the likelihood function $p\left(z_{k}^{1: N} \mid x_{k}^{m}\right)$ as

$$
\begin{aligned}
& p\left(z_{k}^{1: N} \mid x_{k}^{m}\right) \\
= & \prod_{i=1}^{N}\left\{\lambda _ { k } ^ { i } \left[\left(1-\bar{\gamma}^{i}\right) p_{\nu_{k}^{i}+n_{k}^{i}}\left(z_{k}^{i}-h_{k}^{i}\left(x_{k}^{m}\right)\right)\right.\right. \\
& \left.\left.+\bar{\gamma}^{i} p_{\nu_{k}^{i}+n_{k}^{i}}\left(z_{k}^{i}-\operatorname{Sat}\left(h_{k}^{i}\left(x_{k}^{m}\right)\right)\right)\right]+\left(1-\lambda_{k}^{i}\right) p_{n_{k}^{i}}\left(z_{k}^{i}\right)\right\} .
\end{aligned}
$$

Remark 4: Due to the simultaneous presence of the measurement noise and the channel noise, when the energy harvesting sensor $i$ has sufficient energy to send the current measurement to the remote filter, the calculation of the likelihood function depends on the PDF of random variable $\left(\nu_{k}^{i}+n_{k}^{i}\right)$. Noting that $\nu_{k}^{i}$ and $n_{k}^{i}$ are two independent random variables, the PDF of random variable $\left(\nu_{k}^{i}+n_{k}^{i}\right)$ is the convolution of $p_{\nu_{k}^{i}}(\cdot)$ and $p_{n_{k}^{i}}(\cdot)$. To be more specific, if the sensor saturation phenomenon does not occur at time instant $k$, then the likelihood function for the $m$ th particle $x_{k}^{m}$ is calculated by

$p_{\nu_{k}^{i}+n_{k}^{i}}\left(z_{k}^{i}-h_{k}^{i}\left(x_{k}^{m}\right)\right)=\int p_{\nu_{k}^{i}}(\bar{\nu}) p_{n_{k}^{i}}\left(z_{k}^{i}-h_{k}^{i}\left(x_{k}^{m}\right)-\bar{\nu}\right) d \bar{\nu}$.

In fact, some distributions (e.g. normal distribution and exponential distribution) possess simple convolutions. When the accurate convolution is difficult to obtain in some cases, an alternative approximation of (28) is given by a simulation approach. That is,

$$
p_{\nu_{k}^{i}+n_{k}^{i}}\left(z_{k}^{i}-h_{k}^{i}\left(x_{k}^{m}\right)\right) \approx \frac{1}{C} \sum_{c=1}^{C} p_{n_{k}^{i}}\left(z_{k}^{i}-h_{k}^{i}\left(x_{k}^{m}\right)-\bar{\nu}^{c}\right)
$$

where $p_{\nu_{k}^{i}}(\bar{\nu})$ is approximated by its particle representation $\frac{1}{C} \sum_{c=1}^{C} \delta\left(\bar{\nu}-\bar{\nu}^{c}\right)$ and $C$ is the number of the particles.

The implementation of the modified particle filtering algorithm for systems with energy harvesting sensors subject to ROSSs is provided in Algorithm 1. 
Algorithm 1 Modified particle filtering with energy harvesting sensors subject to ROSSs

Step 1. Particle initialization

Sample $M$ particles from the prior density, i.e., $x_{0}^{m} \sim p_{x_{0}}\left(x_{0}\right), m=1,2, \ldots, M$ and the corresponding importance weights $W_{0}^{m}$ are all set to be $\frac{1}{M}$. In addition, set the maximum recursive time instant $K$.

Step 2. Importance sampling

For each $m=1, \ldots, M$, sample particle $x_{k}^{m}$ from the transition PDF $p\left(x_{k}^{m} \mid x_{k-1}^{m}\right)$.

Step 3. Weight update

Calculate the unnormalized weights $\left\{\tilde{W}_{k}^{m}\right\}_{m=1}^{M}$ based on (27) as

$$
\begin{aligned}
& \tilde{W}_{k}^{m} \\
= & W_{k-1}^{m} \prod_{i=1}^{N}\left\{\lambda _ { k } ^ { i } \left[\left(1-\bar{\gamma}^{i}\right) p_{\nu_{k}^{i}+n_{k}^{i}}\left(z_{k}^{i}-h_{k}^{i}\left(x_{k}^{m}\right)\right)\right.\right. \\
& \left.+\bar{\gamma}^{i} p_{\nu_{k}^{i}+n_{k}^{i}}\left(z_{k}^{i}-\operatorname{Sat}\left(h_{k}^{i}\left(x_{k}^{m}\right)\right)\right)\right] \\
& \left.+\left(1-\lambda_{k}^{i}\right) p_{n_{k}^{i}}\left(z_{k}^{i}\right)\right\} .
\end{aligned}
$$

Step 4. Normalization

Normalize the importance weights as

$$
W_{k}^{m}=\frac{\tilde{W}_{k}^{m}}{\sum_{m=1}^{M} \tilde{W}_{k}^{m}} .
$$

Step 5. State estimate update

Calculate the state estimate $\hat{x}_{k}$ and estimation error covariance $P_{k}$ as

$$
\begin{aligned}
\hat{x}_{k} & =\sum_{m=1}^{M} W_{k}^{m} x_{k}^{m}, \\
P_{k} & =\sum_{m=1}^{M} W_{k}^{m}\left(x_{k}^{m}-\hat{x}_{k}\right)\left(x_{k}^{m}-\hat{x}_{k}\right)^{T} .
\end{aligned}
$$

Step 6. Resampling

Resample the particles based on the normalized importance weights $\left\{W_{k}^{m}\right\}_{m=1}^{M}$.

Step 7. If $k<K$, then go to Step 2; otherwise go to Step 8 .

Step 8. Stop.

As can be seen from Algorithm 1, the proposed recursive algorithm is mainly constructed by: 1) sampling new particles from the proposal density function $q\left(x_{k} \mid x_{0: k-1}, Z_{1: k}^{1: N}\right)$ by using previous particles (Steps 1 and 2); and 2) collecting the measurements from all the energy harvesting sensors and updating the importance weights according to the available measurements $z_{k}^{1: N}$, which are dependent on the energy level of each individual sensor and the ROSSs (Steps 3 and 4). For the convenience of implementation, the state transition PDF $p\left(x_{k} \mid x_{k-1}\right)$ determined by (1) is chosen as the proposal density function in Algorithm 1. Nevertheless, after a few iterations, the particle degeneracy phenomenon may occur, which means that all but one particle will have insignificant weights. Therefore, a resampling procedure [3] is introduced to reduce the effect of the degeneracy phenomenon (Step 6). Note that the proposed algorithm can be straightforwardly extended to the adaptive resampling case, i.e., the resampling procedure is executed only when the effective sample size [41] is less than a predefined threshold.

Remark 5: Up to now, the filtering problem has been addressed for a class of nonlinear/non-Gaussian systems subject to the ROSSs and the sensor energy constraints in the framework of sequential Bayesian estimation. By recursively calculating the probability distribution of each sensor's energy level, the probability of measurement transmission is obtained at each time instant. Accordingly, we have derived a modified likelihood function to update the importance weights. It is worth figuring out that our proposed algorithm is also applicable to the case without sensor energy constraints, i.e., $\lambda_{k}^{i}=1$, and the case where the missing measurement is governed by a Bernoulli distributed stochastic variable with known probability distribution [58]. Meanwhile, if we set $\bar{\gamma}^{i}=0$, then our proposed algorithm degenerates to the case with only sensor energy constraints.

Remark 6: It should be pointed out that, in recent years, many particle filtering methods have been proposed to address the model uncertainty by adopting multiple candidate statespace models. A common approach available in the literature is to utilize a batch of particle filters, each of which corresponds to one of the candidate models (see e.g. [5], [9], [29], [30], [42], [55]), and another kind of strategy is to jointly make inferences on the states and model index [10]. Actually, due to the random nature of the sensor saturations and measurement information loss, the measurement process of each sensor can also be described by a system with three candidate models (i.e., $1_{\left\{E_{k}^{i}>0\right\}}=1$ and $\gamma_{k}^{i}=0,1_{\left\{E_{k}^{i}>0\right\}}=1$ and $\gamma_{k}^{i}=1$, and $1_{\left\{E_{k}^{i}>0\right\}}=0$ ) by following the line of [29], [30]. However, it is worth noting that, in this paper, the missing probability of the measurement has been derived in a recursive manner and we are able to directly derive the likelihood function based on the statistical characteristics of the ROSSs and measurement information loss, avoiding the use of multiple particle filters and the inference on the model index.

Remark 7: The particle filtering problem for nonlinear/nonGaussian systems has become a hot topic for a few decades with successful applications in many areas. Comparing to the rich body of existing results in the literature, the main results established in this paper own the following specific merits: 1) the problem addressed is new in the sense that both the energy-dependent transmission protocol and the phenomena of ROSS are taken into account; 2) the particle filtering algorithm proposed is new that tackles the multi-sensor fusion issue subject to partial measurement-information-loss induced by different energy levels of each individual sensor; and 3) the recursion derived for importance weight is new as the joint effects from the ROSSs and the sensor energy constraints are explicitly reflected.

\section{Simulation Results}

In this section, some illustrative examples are presented to verify the effectiveness of our modified particle filtering 


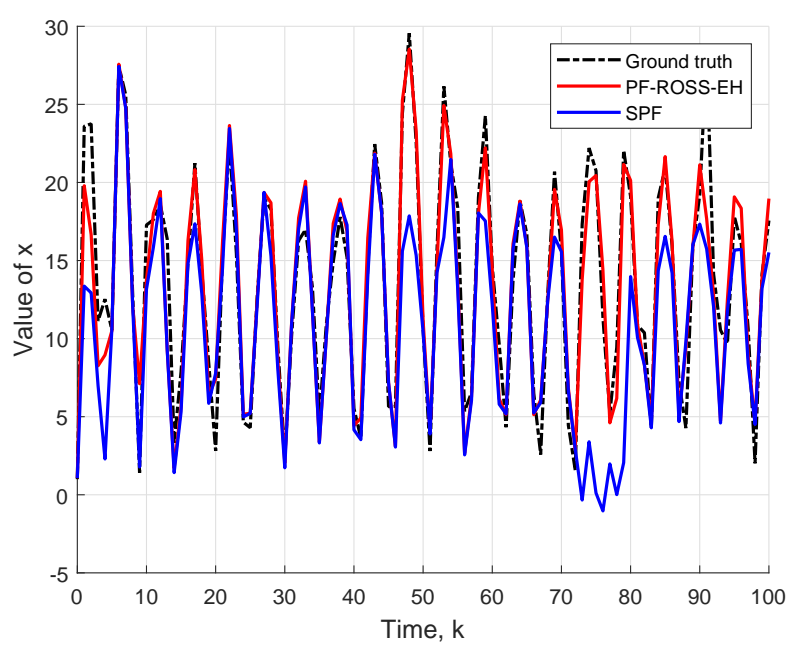

Fig. 2: Estimation results of PF-ROSS-EH and SPF.

algorithm.

\section{A. Numerical example}

In this subsection, a simple non-stationary model (modified from [64], [65]) is employed to show the feasibility of the proposed algorithm (abbreviated as PF-ROSS-EH).

Consider a nonlinear and non-Gaussian system described by (1)-(2) with the following nonlinear functions

$$
\left\{\begin{array}{l}
f_{k}\left(x_{k}\right)=0.5 x_{k}+25 \frac{x_{k}}{1+x_{k}^{2}}+8 \cos (1.2 k) \\
h_{k}^{i}\left(x_{k}\right)=\frac{\left(x_{k}-s_{i}\right)^{2}}{20}, i=1,2
\end{array}\right.
$$

where $s_{1}=0.1$ and $s_{2}=-0.1$. The process noise $\omega_{k}$ satisfies the Gamma distribution, i.e., $\omega_{k} \sim G(2,2)$. The measurement noises $\nu_{k}^{i}$ and channel noises $n_{k}^{i}$ obey the exponential distributions, i.e., $\nu_{k}^{i} \sim \operatorname{Exp}(2)$ and $n_{k}^{i} \sim \operatorname{Exp}(2)$ for $i=1,2$. The occurring probability of the sensor saturation is set to be $\bar{\gamma}^{i}=0.5$ and the saturation level is $\kappa_{\max }=10$. The maximum number of the storable energy for both sensors is $\bar{E}^{i}=3$ and the initial energy is $E_{0}^{i}=1$. The state space of the energy harvesting process is defined as $\mathbb{H}=\{0,1,2,3\}$, and the transition probability matrix is given by

$$
\left[\begin{array}{llll}
\pi_{00} & \pi_{01} & \pi_{02} & \pi_{03} \\
\pi_{10} & \pi_{11} & \pi_{12} & \pi_{13} \\
\pi_{20} & \pi_{21} & \pi_{22} & \pi_{23} \\
\pi_{30} & \pi_{31} & \pi_{32} & \pi_{33}
\end{array}\right]=\left[\begin{array}{llll}
0.5 & 0.2 & 0.2 & 0.1 \\
0.4 & 0.4 & 0.1 & 0.1 \\
0.3 & 0.3 & 0.2 & 0.2 \\
0.3 & 0.1 & 0.2 & 0.4
\end{array}\right] .
$$

In addition, the initial state is $x_{0}=1$ and the initial 200 particles are drawn from a Gaussian prior distribution $N(1,4)$.

For comparison, the standard particle filtering algorithm (abbreviated as SPF) is utilized without considering the ROSSs and the possibly failed measurement transmission. The estimation results are shown in Fig. 2. It can be seen that our PFROSS-EH provides a more accurate estimate than the SPF, which demonstrates the feasibility and effectiveness of our proposed filtering algorithm.

\section{B. Application to 2-D and 3-D target tracking problems}

In this subsection, the proposed algorithm is firstly used to track a target that moves in a two-dimensional plane. The state of the target at time instant $k$ is denoted as

$$
x_{k}=\left[s_{x, k}^{t}, v_{x, k}^{t}, s_{y, k}^{t}, v_{y, k}^{t}\right]^{T},
$$

where $\left(s_{x, k}^{t}, s_{y, k}^{t}\right)$ and $\left(v_{x, k}^{t}, v_{y, k}^{t}\right)$ are the position and velocity of the target's centroid, respectively.

Following [7], the dynamics of the target is represented by the white noise acceleration model described by

$$
x_{k+1}=\left[\begin{array}{cccc}
1 & T & 0 & 0 \\
0 & 1 & 0 & 0 \\
0 & 0 & 1 & T \\
0 & 0 & 0 & 1
\end{array}\right] x_{k}+\omega_{k}
$$

where $T$ denotes the sampling period and $\omega_{k}$ denotes the zeromean Gaussian white noise sequences with covariance matrix $Q_{k}$ determined by

$$
Q_{k}=\Delta\left[\begin{array}{cccc}
\frac{T^{3}}{3} & \frac{T^{2}}{2} & 0 & 0 \\
\frac{T^{2}}{2} & T & 0 & 0 \\
0 & 0 & \frac{T^{3}}{3} & \frac{T^{2}}{2} \\
0 & 0 & \frac{T^{2}}{2} & T
\end{array}\right]
$$

and $\Delta$ denotes the acceleration variance.

Let the moving target emit a radio or acoustic signal. The $N$ passive received-signal-strength (RSS) sensors are deployed in the reconnaissance region to measure the emitted signal energy of the target. To be specific, the measurement model of the $i$ th RSS sensor located at $\left(s_{x, k}^{s, i}, s_{y, k}^{s, i}\right)$ is represented by [7], [46]

$h_{k}^{i}\left(x_{k}\right)=P_{0}-10 n_{r} \log _{10}\left(\frac{\left\|\left[s_{x, k}^{t}, s_{y, k}^{t}\right]^{T}-\left[s_{x, k}^{s, i}, s_{y, k}^{s, i}\right]^{T}\right\|}{d_{0}}\right)$,

$i=1,2, \ldots, N$, where $P_{0}$ is the received signal energy at the reference distance $d_{0}$ and $n_{r}$ is the path loss exponent.

Due to the effect of the ROSSs, the actual measurement output of the $i$ th sensor is given by

$$
y_{k}^{i}=\gamma_{k}^{i} \operatorname{Sat}\left(h_{k}^{i}\left(x_{k}\right)\right)+\left(1-\gamma_{k}^{i}\right) h_{k}^{i}\left(x_{k}\right)+\nu_{k}^{i}
$$

where $\nu_{k}^{i}$ denotes the zero-mean Gaussian white noise sequence with variance $\sigma_{\nu, i}^{2}$. Afterwards, if the sensor energy is sufficient, the measurement output is transmitted to the remote filter via a noisy communication channel (see (7)). The communication noise $n_{k}^{i}$ is modelled by a zero-mean Gaussian white noise sequence with variance $\sigma_{n, i}^{2}$.

To evaluate the performance of the proposed algorithm, the root mean-square error (RMSE) on the position and velocity are respectively defined as follows:

$$
\begin{aligned}
& \operatorname{RMSE}_{\mathrm{Pos}, k}=\sqrt{\frac{1}{M C} \sum_{j=1}^{M C}\left[\left(s_{x, k}^{t, j}-\hat{s}_{x, k}^{t, j}\right)^{2}+\left(s_{y, k}^{t, j}-\hat{s}_{y, k}^{t, j}\right)^{2}\right]}, \\
& \operatorname{RMSE}_{\mathrm{Vel}, k}=\sqrt{\frac{1}{M C} \sum_{j=1}^{M C}\left[\left(v_{x, k}^{t, j}-\hat{v}_{x, k}^{t, j}\right)^{2}+\left(v_{y, k}^{t, j}-\hat{v}_{y, k}^{t, j}\right)^{2}\right]}
\end{aligned}
$$


where $M C$ denotes the total number of the Monte Carlo trials, $\left[s_{x, k}^{t, j}, s_{y, k}^{t, j}\right]^{T}$ and $\left[v_{x, k}^{t, j}, v_{y, k}^{t, j}\right]^{T}$ stand for the realization of $\left[s_{x, k}^{t}, s_{y, k}^{t}\right]^{T}$ and $\left[v_{x, k}^{t}, v_{y, k}^{t}\right]^{T}$, whose estimates in the $j$ th Monte Carlo trial are denoted by $\left[\hat{s}_{x, k}^{t, j}, \hat{s}_{y, k}^{t, j}\right]^{T}$ and $\left[\hat{v}_{x, k}^{t, j}, \hat{v}_{y, k}^{t, j}\right]^{T}$. For the convenience of readers, a summary of the notation involved in this application is provided in TABLE I.

TABLE I: Summary of the involved notation.

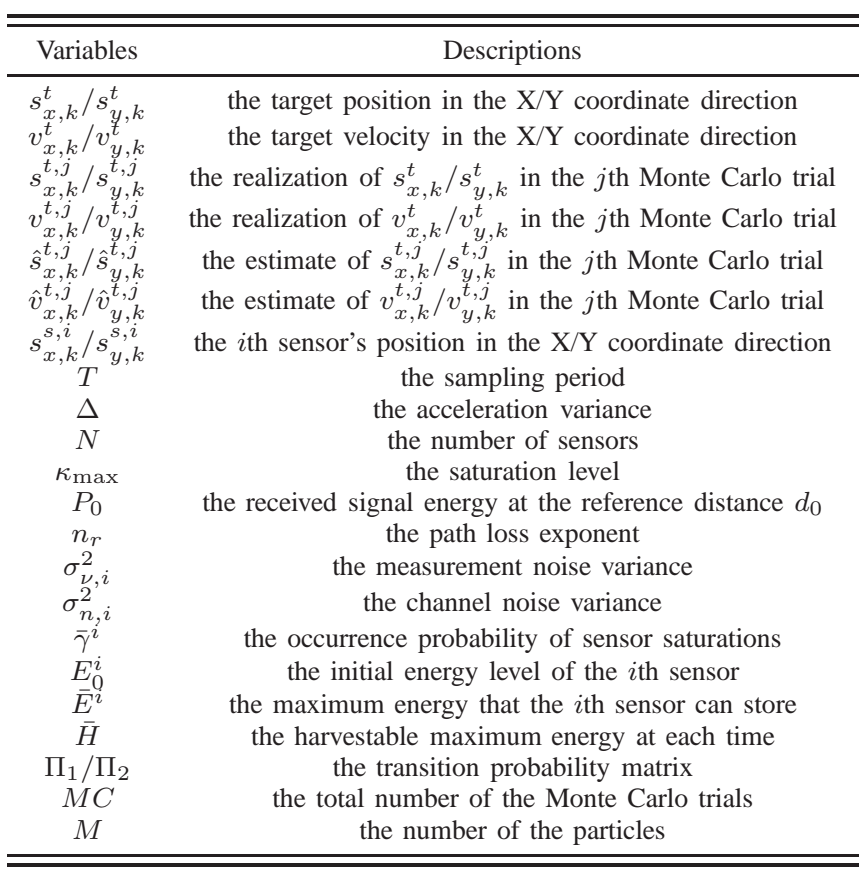

In the simulation, the number of particles is set to be $M=500$ and 100 times of independent Monte Carlo trials are conducted (i.e., $M C=100$ ). In each trial, the true trajectory is generated independently with initial state $x_{0}=\left[\begin{array}{lll}25 \mathrm{~m} & 0.3 \mathrm{~m} / \mathrm{s} \quad 10 \mathrm{~m} \quad 0.4 \mathrm{~m} / \mathrm{s}\end{array}\right]^{T}$. Similar to [7], the position components of the initial particles are drawn from a Gaussian prior distribution with mean $[25,10]^{T}$ and covariance matrix $\operatorname{diag}\left\{25^{2}, 25^{2}\right\}$, while the velocity components are calculated by the resultant velocity and the azimuth which are drawn from a Gaussian prior distribution with mean $\left[\sqrt{(0.3)^{2}+(0.4)^{2}}, \arctan (0.4 / 0.3)\right]^{T}$ and covariance matrix $\operatorname{diag}\left\{0.2^{2},(\pi / 30)^{2}\right\}$. In addition, the state space of the energy harvesting process and the corresponding transition probability matrix are the same as that in Section IV-A. For the sake of clarity, the values of other parameters involved in the simulation are displayed in TABLE II.

The simulation results obtained in one Monte Carlo trial are shown in Figs. 3-5. Fig. 3 sketches the true target trajectory and its corresponding estimate, which shows that our proposed PF-ROSS-EH is able to well track the moving target. Fig. 4 depicts the measurement output of Sensor 4 and the phenomenon of ROSSs while Fig. 5 displays the energy level of Sensor 4 and the transmission instants of measurement output.

For the purpose of comparison, five scenarios, including tracking with our PF-ROSS-EH, tracking with SPF, tracking with standard particle filter using the ideal measurements (unaffected by the ROSSs and the sensor energy constraints,

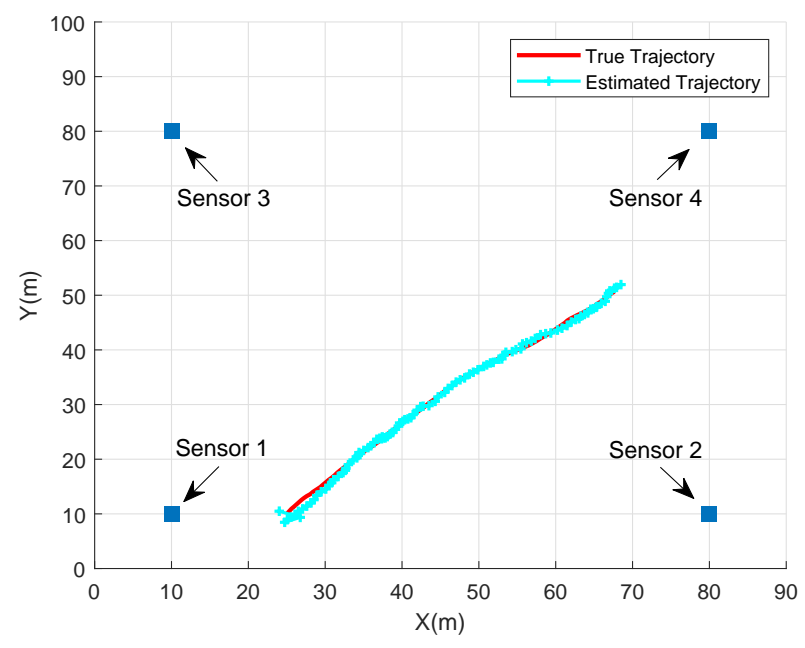

Fig. 3: The true target trajectory and its estimate in one trial.
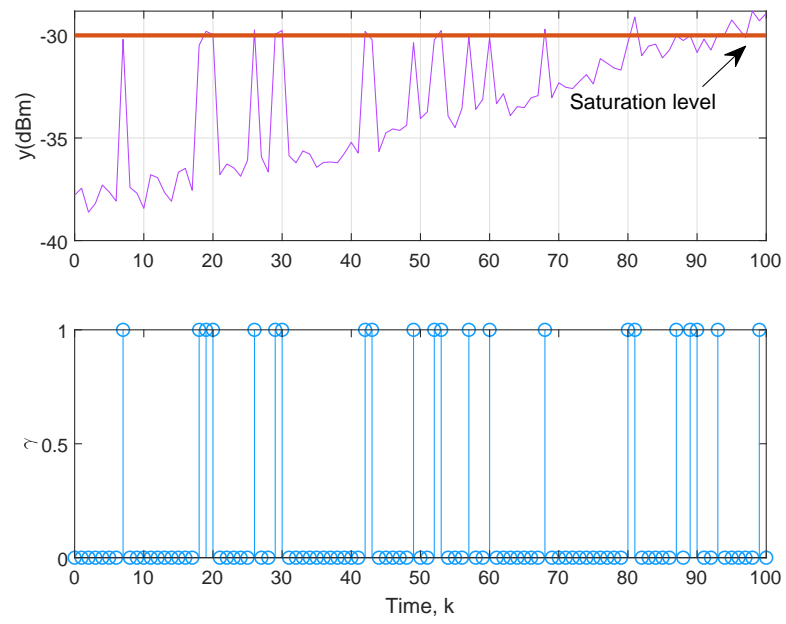

Fig. 4: The measurement output with ROSSs (Sensor 4).
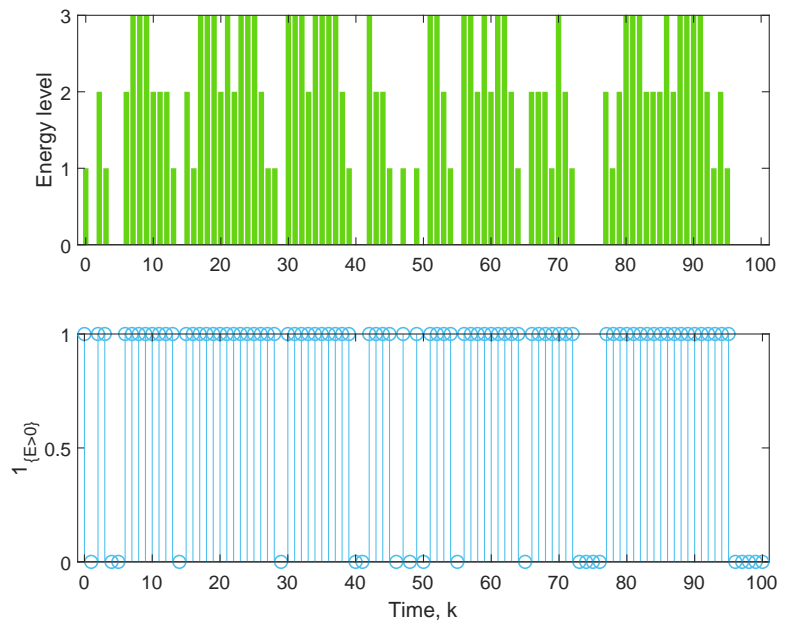

Fig. 5: The energy level and the associated transmission time instants (Sensor 4). 
This article has been accepted for publication in a future issue of this journal, but has not been fully edited. Content may change prior to final publication. Citation information: DOI10.1109/TSP.2020.3042951, IEEE Transactions on Signal Processing

FINAL VERSION

TABLE II: Parameter settings.

\begin{tabular}{lccc}
\hline \hline Parameters & Values & Parameters & Values \\
\hline$T$ & $1 \mathrm{~s}$ & $P_{0}$ & $1 \mathrm{dBm}$ \\
$N$ & 4 & $\kappa_{\max }$ & $30 \mathrm{dBm}$ \\
$d_{0}$ & $1 \mathrm{~m}$ & $\sigma_{\nu, 1}^{2}$ & $0.1 \mathrm{dBm}^{2}$ \\
$n_{r}$ & 2 & $\sigma_{\nu, 2}^{2}$ & $0.049 \mathrm{dBm}^{2}$ \\
$\bar{\gamma}^{i}$ & 0.2 & $\sigma_{\nu, 3}^{2}$ & $0.064 \mathrm{dBm}^{2}$ \\
$E_{0}^{i}$ & 1 & $\sigma_{\nu, 4}^{2}$ & $0.144 \mathrm{dBm}^{2}$ \\
$\bar{E}^{i}$ & 3 & $\sigma_{n, i}^{2}$ & $0.001 \mathrm{dBm}^{2}$ \\
$\bar{H}$ & 3 & $\Delta$ & $0.0016 \mathrm{~m}^{2} / \mathrm{s}^{4}$ \\
\hline \hline
\end{tabular}

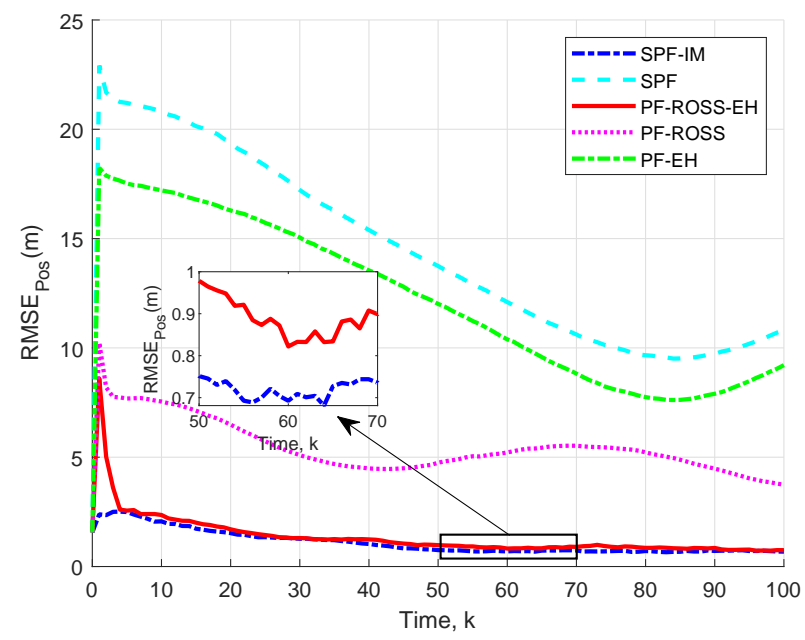

Fig. 6: Position RMSEs of PF-ROSS-EH, PF-ROSS, PF-EH, SPF and SPF-IM.

and abbreviated as SPF-IM), tracking with the particle filter only compensating for the effect of ROSSs (abbreviated as PFROSS), and tracking with the particle filter only compensating for the effect of possibly failed measurement transmission (denoted as PF-EH), will be considered to show the tracking performance. Naturally, we expect that using the unaffected measurements will obtain the best tracking performance among the three scenarios, while neglecting the effect of the above-mentioned phenomena will lead to the worst tracking performance.

The evolutions of the RMSEs on the position estimate and velocity estimate are respectively given in Figs. 6-7. It can be observed from Figs. 6-7 that the tracking performance of our proposed PF-ROSS-EH approaches to that of the SPFIM and is much better than that of the SPF, PF-ROSS, and PF-EH. This is reasonable since we have made much effort to compensate for the effect of the ROSSs and the possible missing measurements.

Next, we will conduct further simulations with different occurrence probabilities of the sensor saturations, different numbers of sensors and different transition probability matrices in the energy harvesting process to analyze their respective effect on the tracking performance. The simulation results with regard to different occurrence probabilities of the sensor saturations (i.e., $\bar{\gamma}^{i}=0.2,0.5,0.8$ ) and different numbers

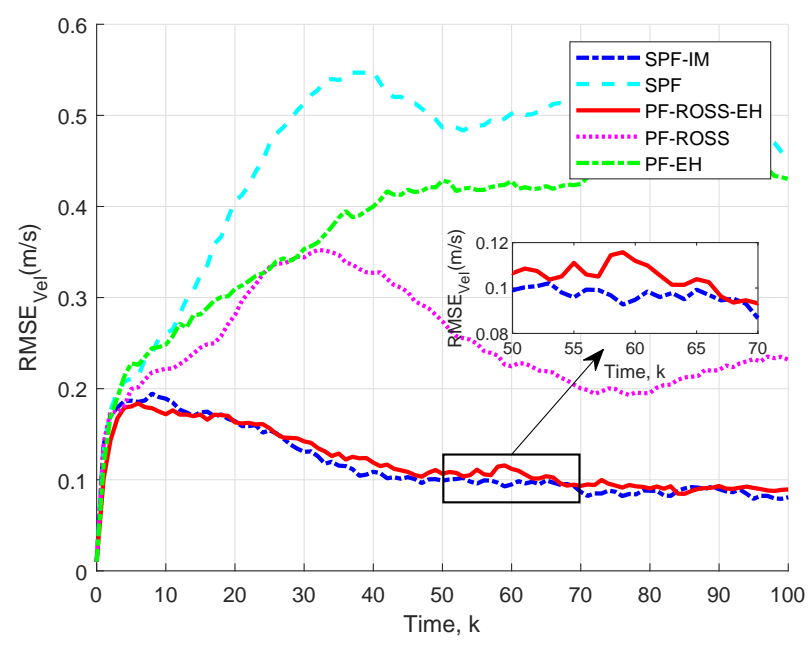

Fig. 7: Velocity RMSEs of PF-ROSS-EH, PF-ROSS, PF-EH, SPF and SPF-IM.
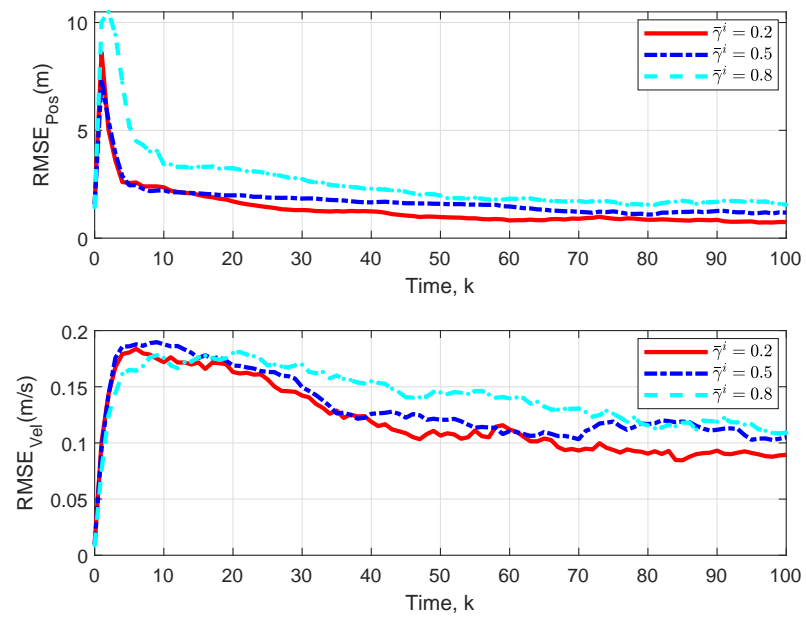

Fig. 8: Position and velocity RMSEs of PF-ROSS-EH with different occurrence probabilities of the sensor saturations.

of sensors (i.e., $N=2,4,6$ ) are, respectively, displayed in Figs. 8-9, which show that the occurrence probability of the random sensor saturations and the number of sensors both have notable effects on the tracking performance. Specifically, the tracking performance degrades with the increase of the occurrence probability of sensor saturations, and improves as the number of sensors increases. On the other hand, we denote the transition probability matrix used before as $\Pi_{1}$ and choose another transition probability matrix $\Pi_{2}$ as

$$
\Pi_{2}=\left[\begin{array}{cccc}
0.8 & 0.1 & 0.05 & 0.05 \\
0.7 & 0.1 & 0.1 & 0.1 \\
0.6 & 0.2 & 0.1 & 0.1 \\
0.5 & 0.2 & 0.2 & 0.1
\end{array}\right] .
$$

The corresponding simulation results are illustrated in Fig. 10. From Fig. 10, we can see that the tracking performance of the proposed algorithm with transition probability matrix $\Pi_{1}$ is superior to that with $\Pi_{2}$, which implies that the energy 

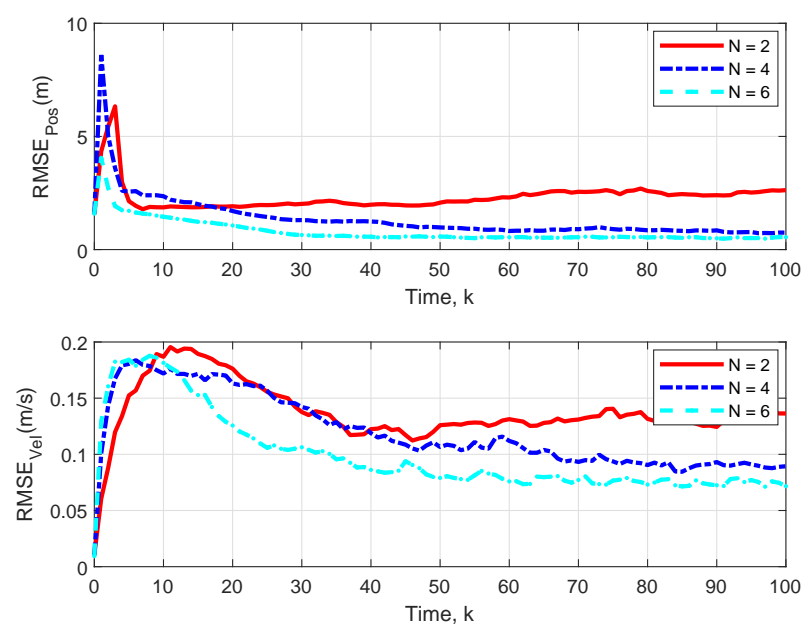

Fig. 9: Position and velocity RMSEs of PF-ROSS-EH with different numbers of sensors.
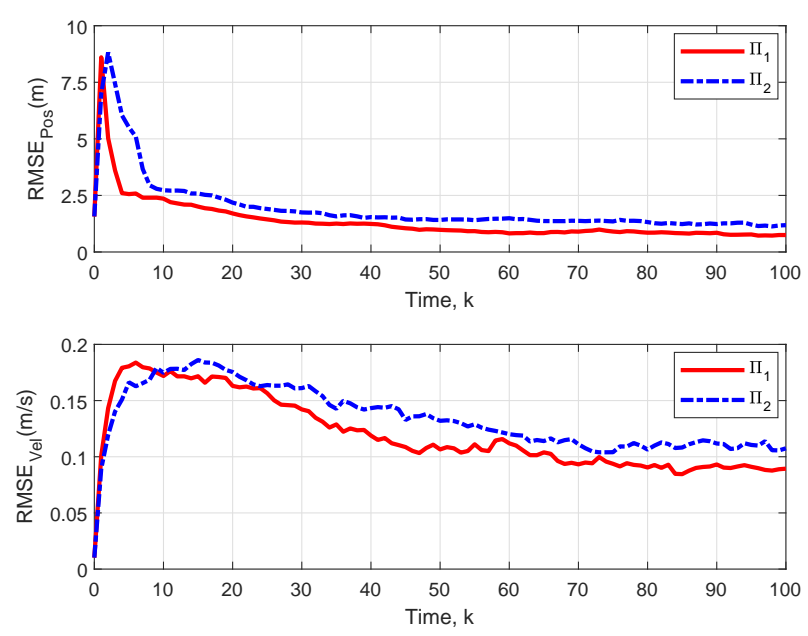

Fig. 10: Position and velocity RMSEs of PF-ROSS-EH with different energy harvesting processes.

harvesting process has a non-negligible effect on the filtering accuracy. In fact, the results are intuitively reasonable because the energy harvesting process with transition probability matrix $\Pi_{2}$ is more likely to harvest zero energy and is easier to incur missing measurement.

In what follows, the 3-D moving target tracking scenario is considered to further demonstrate the effectiveness of the proposed algorithm. Similarly, the dynamics of the target is represented by the white noise acceleration model [4] and the state of the target at time instant $k$ is represented by

$$
x_{k}=\left[s_{x, k}^{t}, v_{x, k}^{t}, s_{y, k}^{t}, v_{y, k}^{t}, s_{z, k}^{t}, v_{z, k}^{t}\right]^{T},
$$

where $\left(s_{x, k}^{t}, s_{y, k}^{t}, s_{z, k}^{t}\right)$ and $\left(v_{x, k}^{t}, v_{y, k}^{t}, v_{z, k}^{t}\right)$ are the position and velocity of the target's centroid, respectively. The measurement model of the $i$ th RSS sensor is based on the distance between the moving target and the $i$ th sensor in the 3-D scenario. The RMSEs on the position and

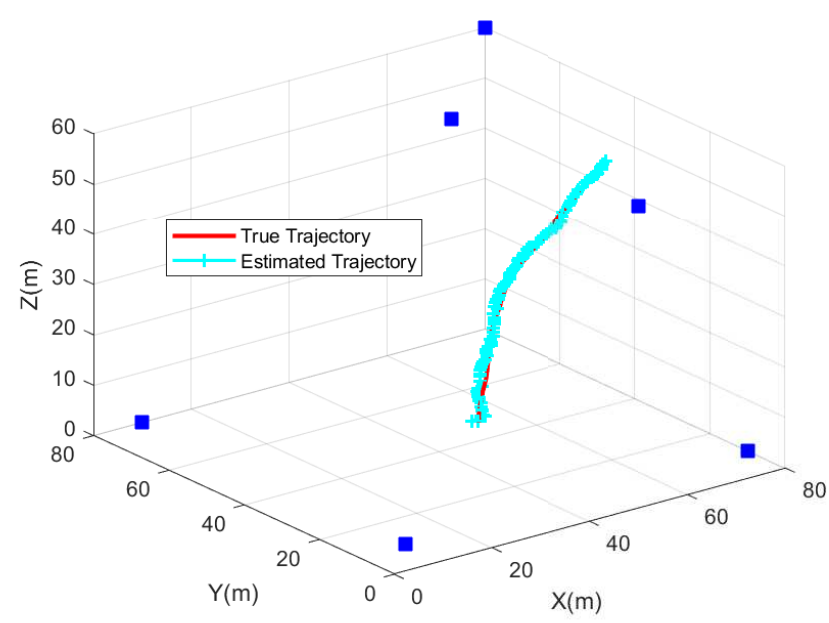

Fig. 11: The true target trajectory and its estimate in the 3-D scenario.

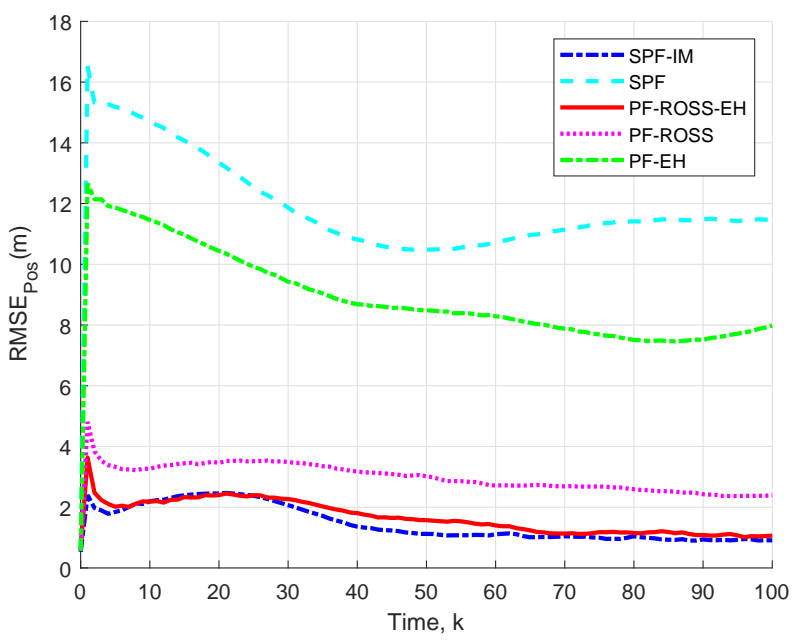

Fig. 12: Position RMSEs of PF-ROSS-EH, PF-ROSS, PF-EH, SPF and SPF-IM in the 3-D scenario.

velocity are similarly defined. The initial state is chosen

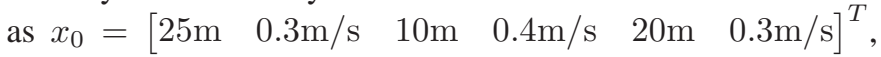
and the initial particles are sampled from a Gaussian prior distribution with mean $x_{0}$ and covariance $\operatorname{diag}\left\{10^{2}, 0.3^{2}, 10^{2}, 0.3^{2}, 10^{2}, 0.1^{2}\right\}$. In the simulation, the number of sensors is set as $N=6$ and the number of particles is $M=1000$. Other parameters are the same as those in the 2-D scenario.

The simulation results in the 3-D scenario are displayed in Figs. 11-13, which again verify the effectiveness of the proposed particle filtering algorithm in the simultaneous presence of the ROSSs and possibly failed measurement transmission.

\section{Conclusions}

In this paper, a particle filtering algorithm has been developed to solve the filtering problem for a class of nonlinear/non- 


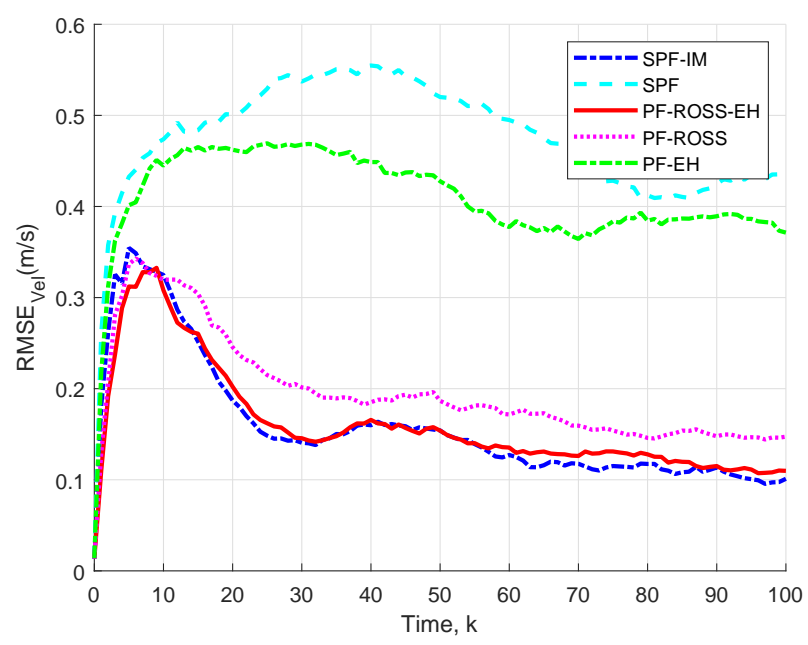

Fig. 13: Velocity RMSEs of PF-ROSS-EH, PF-ROSS, PF-EH, SPF and SPF-IM in the 3-D scenario.

Gaussian systems with energy harvesting sensors subject to the ROSSs. According to the established first-order Markov model of the energy harvesting process, the occurrence probability of the missing measurement has been calculated at each time instant. A modified likelihood function, which takes the occurrence probability of the ROSSs and the missing probability of the measurements induced by insufficient energy into consideration, has been derived to attenuate the effects from both ROSSs and sensor energy constraints on the filtering quality. Finally, the usefulness of the proposed filtering scheme has been demonstrated by the illustrative examples. Future research topics would be the extensions of the present results to more sophisticated energy harvesting models (e.g. nonMarkovian model), and to distributed state estimation problem with large datasets [36], [48], [60], in which the herding algorithms [19], the compressed Monte Carlo schemes [39] and the group importance sampling schemes [40] might be considered to reduce the computational burden and the communication cost.

\section{REFERENCES}

[1] B. D. O. Anderson and J. B. Moore, Optimal Filtering. Englewood Cliffs, NJ, USA: Prentice-Hall, 1979.

[2] I. Arasaratnam and S. Haykin, Cubature Kalman filters, IEEE Transactions on Automatic Control, vol. 54, no. 6, pp. 1254-1269, 2009.

[3] M. S. Arulampalam, S. Maskell, N. Gordon and T. Clapp, A tutorial on particle filters for online nonlinear/non-Gaussian Bayesian tracking, IEEE Transactions on Signal Processing, vol. 50, no. 2, pp. 174-188, 2002.

[4] Y. Bar-Shalom, X. R. Li and T. Kirubarajan, Estimation with Applications to Tracking and Navigation. New York: Wiley, 2001.

[5] Y. Boers and J. N. Driessen, Interacting multiple model particle filter, IEE Proceedings-Radar, Sonar and Navigation, vol. 150, no. 5, pp. 344 349, 2003.

[6] Y. Chen, Z. Wang, L. Wang and W. Sheng, Finite-horizon $H_{\infty}$ state estimation for stochastic coupled networks with random inner couplings using Round-Robin protocol, IEEE Transactions on Cybernetics, in press, DOI: 10.1109/TCYB.2020.3004288.

[7] S. S. Dias and M. G. S. Bruno, Cooperative target tracking using decentralized particle filtering and RSS sensors, IEEE Transactions on Signal Processing, vol. 61, no. 14, pp. 3632-3646, 2013.
[8] D. Ding, Z. Wang, B. Shen and H. Shu, $H_{\infty}$ state estimation for discrete-time complex networks with randomly occurring sensor saturations and randomly varying sensor delays, IEEE Transactions on Neural Networks and Learning Systems, vol. 23, no. 5, pp. 725-736, 2012.

[9] C. C. Drovandi, J. M. McGree and A. N. Pettitt, A sequential Monte Carlo algorithm to incorporate model uncertainty in Bayesian sequential design, Journal of Computational and Graphical Statistics, vol. 23, no. 1, pp. 3-24, 2014.

[10] Y. El-Laham, L. Yang, P. M. Djurić and M. F. Bugallo, Particle filtering under general regime switching, arXiv: 2009.04551, 2020.

[11] H. Geng, Z. Wang and Y. Cheng, Distributed federated Tobit Kalman filter fusion over a packet-delaying network: a probabilistic perspective, IEEE Transactions on Signal Processing, vol. 66, no. 17, pp. 4477-4489, 2018.

[12] N. J. Gordon, D. J. Salmond and A. F. M. Smith, Novel approach to nonlinear/non-Gaussian Bayesian state estimation, IEE Proceedings $F$ (Radar and Signal Processing), vol. 140, no. 2, pp. 107-113, 1993.

[13] C. K. Ho, P. D. Khoa and P. C. Ming, Markovian models for harvested energy in wireless communications, in Proceedings of IEEE International Conference on Communication Systems, Singapore, 2010, pp. 311315.

[14] C. K. Ho and R. Zhang, Optimal energy allocation for wireless communications with energy harvesting constraints, IEEE Transactions on Signal Processing, vol. 60, no. 9, pp. 4808-4818, 2012.

[15] Y. C. Ho and R. C. K. Lee, A Bayesian approach to problems in stochastic estimation and control, IEEE Transactions on Automatic Control, vol. 9, no. 4, pp. 333-339, 1964.

[16] J. Hu, Z. Wang and H. Gao, Joint state and fault estimation for timevarying nonlinear systems with randomly occurring faults and sensor saturations, Automatica, vol. 97, pp. 150-160, 2018.

[17] J. Hu, Z. Wang, G.-P. Liu, H. Zhang and R. Navaratne, A predictionbased approach to distributed filtering with missing measurements and communication delays through sensor networks, IEEE Transactions on Systems, Man, and Cybernetics-Systems, in press, DOI: 10.1109/TSMC. 2020.2966977 .

[18] J. Huang, D. Shi and T. Chen, Event-triggered state estimation with an energy harvesting sensor, IEEE Transactions on Automatic Control, vol. 62 , no. 9 , pp. $4768-4775,2017$.

[19] F. Huszár and D. Duvenaud, Optimally-weighted herding is Bayesian quadrature, in Proceedings of the Twenty-Eighth Conference on Uncertainty in Artificial Intelligence, Catalina Island, CA, 2012, pp. 377-386.

[20] S. Julier and J. Uhlmann, Unscented filtering and nonlinear estimation, Proceeding of the IEEE, vol. 92, no. 3, pp. 401-422, 2004.

[21] S. Julier, J. Uhlmann and H. F. Durrant-Whyte, A new method for the nonlinear transformation of means and covariances in filters and estimators, IEEE Transactions on Automatic Control, vol. 45, no. 3 , pp. 477-482, 2000.

[22] S. Knorn, S. Dey, A. Ahlén and D. E. Quevedo, Optimal energy allocation in multisensor estimation over wireless channels using energy harvesting and sharing, IEEE Transactions on Automatic Control, vol. 64, no. 10, pp. 4337-4344, 2019.

[23] A. S. Leong, S. Dey and D. E. Quevedo, Transmission scheduling for remote state estimation and control with an energy harvesting sensor, Automatica, vol. 91, pp. 54-60, 2018.

[24] J. Li, Z. Wang, H. Dong and F. Han, Delay-distribution-dependent state estimation for neural networks under stochastic communication protocol with uncertain transition probabilities, Neural Networks, vol. 130, pp. 143-151, Oct. 2020.

[25] Q. Li, Z. Wang, N. Li and W. Sheng, A dynamic event-triggered approach to recursive filtering for complex networks with switching topologies subject to random sensor failures, IEEE Transactions on Neural Networks and Learning Systems, Vol. 31, No. 10, pp. 4381-4388, Oct. 2020.

[26] Q. Li, Z. Wang, W. Sheng, F. E. Alsaadi and F. E. Alsaadi, Dynamic event-triggered mechanism for $H_{\infty}$ non-Fragile state estimation of complex networks under randomly occurring sensor saturations, Information Sciences, vol. 509, pp. 304-316, Jan. 2020.

[27] W. Li, Z. Wang, Q. Liu and L. Guo, An information aware eventtriggered scheme for particle filter based remote state estimation, Automatica, vol. 103, pp. 151-158, 2019.

[28] W. Li, Z. Wang, Y. Yuan and L. Guo, Particle filtering with applications in networked systems: a survey, Complex \& Intelligent Systems, vol. 2, no. 4, pp. 293-315, 2016.

[29] B. Liu, Instantaneous frequency tracking under model uncertainty via dynamic model averaging and particle filtering, IEEE Transactions on Wireless Communications, vol. 10, no. 6, pp. 1810-1819, 2011. 
[30] B. Liu, Robust particle filter by dynamic averaging of multiple noise models, in Proceedings of 2017 IEEE International Conference on Acoustics, Speech and Signal Processing (ICASSP), New Orleans, LA, 2017, pp. 4034-4038.

[31] D. Liu, Z. Wang, Y. Liu and F. E. Alsaadi, Extended Kalman filtering subject to random transmission delays: Dealing with packet disorders, Information Fusion, vol. 60, pp. 80-86, Aug. 2020.

[32] Q. Liu and Z. Wang, Moving-horizon estimation for linear dynamic networks with binary encoding schemes, IEEE Transactions on Automatic Control, in press, DOI: 10.1109/TAC.2020.2996579.

[33] S. Liu, Z. Wang, Y. Chen and G. Wei, Protocol-based unscented Kalman filtering in the presence of stochastic uncertainties, IEEE Transactions on Automatic Control, vol. 65, no. 3, pp. 1303-1309, Mar. 2020.

[34] Y. Liu, Z. Wang, X. He and D. H. Zhou, Event-triggered filtering and fault estimation for nonlinear systems with stochastic sensor saturations, International Journal of Control, vol. 90, no. 5, pp. 1052-1062, 2017.

[35] Y. Liu, Z. Wang, Y. Yuan and W. Liu, Event-triggered partial-nodesbased state estimation for delayed complex networks with bounded distributed delays, IEEE Transactions on Systems, Man, and CyberneticsSystems, vol. 49, no. 6, pp. 1088-1098, Jun. 2019.

[36] D. Luengo, L. Martino, V. Elvira and M. Bugallo, Efficient linear fusion of partial estimators, Digital Signal Processing, vol. 78, pp. 265-283, 2018.

[37] L. Ma, Z. Wang, H.-K. Lam and N. Kyriakoulis, Distributed event-based set-membership filtering for a class of nonlinear systems with sensor saturations over sensor networks, IEEE Transactions on Cybernetics, vol. 47, no. 11, pp. 3772-3783, 2017.

[38] L. Ma, Z. Wang, C. Cai and F. E. Alsaadi, A dynamic event-triggered approach to $H_{\infty}$ control for discrete-time singularly perturbed systems with time-delays and sensor saturations, IEEE Transactions on Systems, Man, and Cybernetics-Systems, in press, DOI: 10.1109/TSMC.2019.2958529.

[39] L. Martino and V. Elvira, Compressed Monte Carlo for distributed Bayesian inference, viXra: 1811.0505, 2018.

[40] L. Martino, V. Elvira, G. Camps-Valls, Group importance sampling for particle filtering and MCMC, Digital Signal Processing, vol. 82, pp. 133-151, 2018.

[41] L. Martino, V. Elvira and F. Louzada, Effective sample size for importance sampling based on discrepancy measures, Signal Processing, vol. 131, pp. 386-401, 2017.

[42] L. Martino, J. Read, V. Elvira and F. Louzada, Cooperative parallel particle filters for online model selection and applications to urban mobility, Digital Signal Processing, vol. 60, pp. 172-185, 2017.

[43] J. Míguez, Analysis of selection methods for cost-reference particle filtering with applications to maneuvering target tracking and dynamic optimization, Digital Signal Processing, vol. 17, no. 4, pp. 787-807, 2007.

[44] D. Niyato, E. Hossain, M. M. Rashid and V. K. Bhargava, Wireless sensor networks with energy harvesting technologies: a game-theoretic approach to optimal energy management, IEEE Wireless Communications, vol. 14, no. 4, pp. 90-96, 2007.

[45] M. Orton and A. Marrs, Particle filters for tracking with out-of-sequence measurements, IEEE Transactions on Aerospace and Electronic Systems, vol. 41, no. 2, pp. 693-702, 2005.

[46] N. Patwari, A. O. Hero III, M. Perkins, N. S. Correal and R. J. O'Dea, Relative location estimation in wireless sensor networks, IEEE Transactions on Signal Processing, vol. 51, no. 8, pp. 2137-2148, 2003.

[47] W. Qian, Y. Li, Y. Chen, and W. Liu, $L_{2}-L_{\infty}$ filtering for stochastic delayed systems with randomly occurring nonlinearities and sensor saturation, International Journal of Systems Science, in press, DOI: 10.1080/00207721.2020.1794080.

[48] S. L. Scott, A. W. Blocker, F. V. Bonassi, H. A. Chipman, E. I. George and R. E. McCulloch, Bayes and big data: the consensus Monte Carlo algorithm, International Journal of Management Science and Engineering Management, vol. 11, no. 2, pp. 78-88, 2016.

[49] B. Shen, Z. Wang, H. Shu and G. Wei, On nonlinear H-infinity filtering for discrete-time stochastic systems with missing measurements, IEEE Transactions on Automatic Control, vol. 53, no. 9, pp. 2170-2180, 2008.

[50] B. Shen, Z. Wang, D. Wang, J. Luo, H. Pu and Y. Peng, Finite-horizon filtering for a class of nonlinear time-delayed systems with an energy harvesting sensor, Automatica, vol. 100, pp. 144-152, 2019.

[51] B. Shen, Z. Wang, D. Wang and Q. Li, State-saturated recursive filter design for stochastic time-varying nonlinear complex networks under deception attacks, IEEE Transactions on Neural Networks and Learning Systems, Vol. 31, No. 10, pp. 3788-3800, Oct. 2020.

[52] Y. Shen, Z. Wang, B. Shen and F. E. Alsaadi, $H_{\infty}$ filtering for multi-rate multi-sensor systems with randomly occurring sensor saturations under the p-persistent CSMA protocol, IET Control Theory and Applications, vol. 14, no. 10, pp. 1255-1265, Jul. 2020.

[53] Y. Shen, Z. Wang, B. Shen, F. E. Alsaadi and F. E. Alsaadi, Fusion estimation for multi-rate linear repetitive processes under weighted Try-Once-Discard protocol, Information Fusion, vol. 55, pp. 281-291, Mar. 2020.

[54] S. Sudevalayam and P. Kulkarni, Energy harvesting sensor nodes: survey and implications, IEEE Communications Surveys \& Tutorials, vol. 13, no. 3, pp. 443-461, 2011.

[55] I. Urteaga, M. F. Bugallo and P. M. Djurić, Sequential Monte Carlo methods under model uncertainty, in Proceedings of 2016 IEEE Statistical Signal Processing Workshop (SSP), Spain, 2016, pp. 1-5.

[56] D. Wang, Z. Wang, B. Shen and F. E. Alsaadi, Security-guaranteed filtering for discrete-time stochastic delayed systems with randomly occurring sensor saturations and deception attacks, International Journal of Robust and Nonlinear Control, vol. 27, no. 7, pp. 1194-1208, 2017.

[57] M. Wang, Z. Wang, Y. Chen and W. Sheng, Adaptive neural eventtriggered control for discrete-time strict-feedback nonlinear systems, IEEE Transactions on Cybernetics, vol. 50, no. 7, pp. 2946-2958, Jul. 2020.

[58] Z. Wang, B. Shen and X. Liu, $H_{\infty}$ filtering with randomly occurring sensor saturations and missing measurements, Automatica, vol. 48 , no. 3 , pp. 556-562, 2012.

[59] J. Wu, Q.-S. Jia, K. H. Johansson and L. Shi, Event-based sensor data scheduling: trade-off between communication rate and estimation quality, IEEE Transactions on Automatic Control, vol. 58, no. 4, pp. 10411046, 2013.

[60] J.-J. Xiao, A. Ribeiro, Z.-Q. Luo and G. B. Giannakis, Distributed compression-estimation using wireless sensor networks, IEEE Signal Processing Magazine, vol. 23, no. 4, pp. 27-41, 2006.

[61] L. Xu, K. Ma, W. Li, Y. Liu and F. E. Alsaadi, Particle filtering for networked nonlinear systems subject to random one-step sensor delay and missing measurements, Neurocomputing, vol. 275, pp. 2162-2169, 2018.

[62] C. Yang and H. Fang, Modified particle filter and Gaussian filter with packet dropouts, International Journal of Robust and Nonlinear Control, vol. 28, no. 8, pp. 2961-2975, 2018.

[63] X.-M. Zhang, Q.-L. Han, X. Ge, D. Ding, L. Ding, D. Yue and C. Peng, Networked control systems: A survey of trends and techniques, IEEECAA Journal of Automatica Sinica, vol. 7, no. 1, pp. 1-17, Jan. 2020.

[64] Y. Zhang, Y. Huang, N. Li and L, Zhao, Particle filter with onestep randomly delayed measurements and unknown latency probability, International Journal of Systems Science, vol. 47, no. 1, pp. 209-221, 2016.

[65] J. Zuo and X. Zhong, Particle filter for nonlinear systems with multisensor asynchronous random delays, Journal of Systems Engineering and Electronics, vol. 28, no. 6, pp. 1064-1071, 2017.

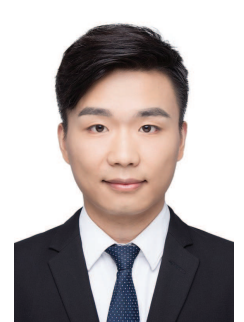

Weihao Song received the B.S. degree in flight vehicle design and engineering from Beijing Institute of Technology, Beijing, China, in 2016. He is currently pursuing the $\mathrm{Ph} . \mathrm{D}$. degree in aeronautical and astronautical science and technology with Beijing Institute of Technology, Beijing, China.

From May 2019 to May 2020, he was a Visiting Scholar with the Department of Computer Science, Brunel University London, London, U.K. His research interests include Bayesian state estimation, distributed state estimation, nonlinear filtering, and networked control systems. 
This article has been accepted for publication in a future issue of this journal, but has not been fully edited. Content may change prior to final publication. Citation information: DOI10.1109/TSP.2020.3042951, IEEE Transactions on Signal Processing

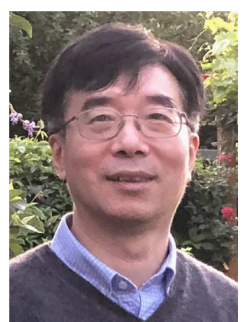

Zidong Wang (SM'03-F'14) was born in Jiangsu, China, in 1966. He received the B.Sc. degree in mathematics in 1986 from Suzhou University, Suzhou, China, and the M.Sc. degree in applied mathematics in 1990 and the Ph.D. degree in electrical engineering in 1994, both from Nanjing University of Science and Technology, Nanjing, China.

$\mathrm{He}$ is currently a Professor of Dynamical Systems and Computing in the Department of Computer Science, Brunel University London, U.K. From 1990 to 2002, he held teaching and research appointments in universities in China, Germany and the UK. Prof. Wang's research interests include dynamical systems, signal processing, bioinformatics, control theory and applications. He has published around 600 papers in refereed international journals. He is a holder of the Alexander von Humboldt Research Fellowship of Germany, the JSPS Research Fellowship of Japan, William Mong Visiting Research Fellowship of Hong Kong.

Prof. Wang serves (or has served) as the Editor-in-Chief for Neurocomputing, Deputy Editor-in-Chief for International Journal of Systems Science, and an Associate Editor for 12 international journals including IEEE Transactions on Automatic Control, IEEE Transactions on Control Systems Technology, IEEE Transactions on Neural Networks, IEEE Transactions on Signal Processing, and IEEE Transactions on Systems, Man, and Cybernetics-Part C. $\mathrm{He}$ is a Member of the Academia Europaea, a Fellow of the IEEE, a Fellow of the Royal Statistical Society and a member of program committee for many international conferences.

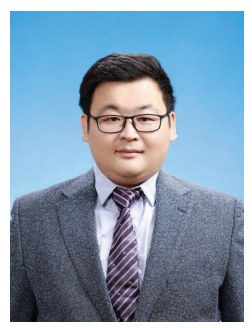

Jianan Wang (Senior Member, IEEE) received the B.S. and M.S. degrees in control theory and engineering from Beijing Jiaotong University and Beijing Institute of Technology, Beijing, China, in 2004 and 2007, respectively. He received the Ph.D. degree in aerospace engineering from Mississippi State University, Starkville, MS, USA, in 2011.

$\mathrm{He}$ is currently an Associate Professor with the School of Aerospace Engineering, Beijing Institute of Technology. His research interests include cooperative control of multiple dynamic systems, UAV formation control, cooperative guidance, and estimation of sensor networks. $\mathrm{He}$ is a senior member of IEEE and AIAA.

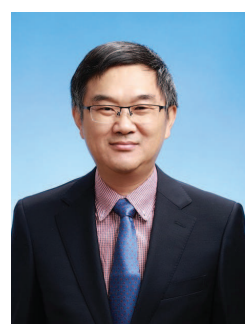

Jiayuan Shan received the B.S. degree from Huazhong University of Science and Technology, Wuhan, China, in 1988, and the M.S. and Ph.D. degrees from Beijing Institute of Technology, Beijing, China, in 1991 and 1999, respectively.

$\mathrm{He}$ is currently a Professor with the School of Aerospace Engineering, Beijing Institute of Technology. His research interests include guidance, navigation and control of the aircraft and hardware-in-theloop simulation. He is the Principal Professor in the direction of Flight Dynamics and Control.

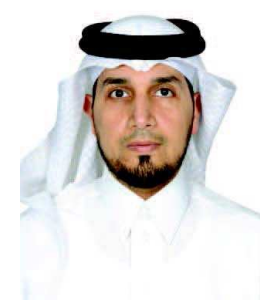

Fuad E. Alsaadi received the B.S. and M.Sc degrees in electronic and communication from King AbdulAziz University, Jeddah, Saudi Arabia, in 1996 and 2002. He then received the Ph.D. degree in Optical Wireless Communication Systems from the University of Leeds, Leeds, UK, in 2011. Between 1996 and 2005, he worked in Jeddah as a communication instructor in the College of Electronics \& Communication. He is currently an associate professor of the Electrical and Computer Engineering Department within the Faculty of Engineering, King Abdulaziz University, Jeddah, Saudi Arabia. He published widely in the top IEEE communications conferences and journals and has received the Carter award, University of Leeds for the best $\mathrm{PhD}$. He has research interests in optical systems and networks, signal processing, synchronization and systems design. 